Research Article

\title{
Robust System Design Using BILP for Wireless Indoor Positioning Systems
}

\author{
Kriangkrai Maneerat $(\mathbb{D})$ and Kamol Kaemarungsi (iD \\ National Electronics and Computer Technology Center, NSTDA, Pathumthani, Thailand \\ Correspondence should be addressed to Kamol Kaemarungsi; kamol.kaemarungsi@nectec.or.th
}

Received 27 October 2017; Revised 17 January 2018; Accepted 31 January 2018; Published 25 March 2018

Academic Editor: Joaquín Torres-Sospedra

Copyright (c) 2018 Kriangkrai Maneerat and Kamol Kaemarungsi. This is an open access article distributed under the Creative Commons Attribution License, which permits unrestricted use, distribution, and reproduction in any medium, provided the original work is properly cited.

In wireless indoor positioning system designs, reference node $(\mathrm{RN})$ failures during the online phase cause received signal strength values to be unavailable. This leads to accuracy performance degradation and a lack of system reliability in smart office systems. Moreover, the major design concern in the reliability of indoor positioning systems under the faulty RNs during the online phase has not been yet investigated in previous works. To address these gaps, we propose a novel mathematical formulation using a Binary Integer Linear Programming (BILP) approach that employs the Simulated Annealing (SA) solution technique. The proposed robust system design aims to put in place a suitable number of RNs and to determine their optimum locations, which may be located on a single floor or on multiple floors. In particular, the proposed system design provisions to support robust operation both during a normal situation and when there are some RN failures. Experimental results and comparative performance evaluation revealed that the proposed robust system design outperformed other system designs and was able to achieve the highest location accuracy performance in both fault-free and $\mathrm{RN}$-failure scenarios. Specifically, when nine of the RNs in a three-story building failed, the proposed system design achieved $84.6 \%, 54.7 \%$, and $32.9 \%$ more accurate performance than the Uniform, the MSMR, and the PhI-Uni, respectively.

\section{Introduction}

The rapid growth of wireless communication technologies is the major driving force behind the integration of the various embedded devices and systems in the vision of smart cities. The systems in a smart city deploy a combination of data collection, processing, and allocation technologies in conjunction with network and computer technologies. These provide several advantages, such as an effective resource allocation as well as higher data security and privacy, facilitating the development of smart cities and enhancing the quality of life for their citizens [1]. One of the applications currently deployed for smart cities is location-based services. For example, Li et al.[2] presented a wireless indoor positioning system that can monitor and track the location of the visitors in a smart office building. Nazari et al. [3] proposed the development of a hospital solution framework for smart health systems, in which the staff can quickly check the location and availability of the needed equipment in an emergency situation. Wang et al. [4] presented indoor location services for smart shopping systems, which enable customers to ask the system to find the shortest path for them to obtain the desired product in a supermarket. Therefore, this article focuses on indoor positioning systems inside smart buildings and smart offices that can be considered as important parts of smart cities.

A wireless indoor positioning system is one of the key capabilities of context-aware computing, allowing the system to determine the location of a mobile station inside a building. The physical information of a mobile station with respect to a set of reference positions within a service area is analyzed and used to estimate its location by using a localization algorithm. Thus, the challenges of indoor positioning systems in terms of location estimation are not only to achieve performance accuracy in finding the required location, but also to overcome completely various indoor positioning issues, such as the problems arising from a dense indoor environment (e.g., multipath effect) [5], technology 
capabilities (e.g., hardware limitations) [6], and the types of service areas (e.g., inside multistory buildings) [7]. Generally, the operation of a wireless indoor positioning system is divided into two processes: the system design phase and the localization phase.

First, in the system design phase, various wireless network configurations are considered and determined by the system designer. These are provisioned to support the indoor positioning system before implementing and deploying the actual localization system. This is a necessary and indeed essential process for an indoor positioning system to achieve any required performance goal. An example of wireless network configurations that are considered in the system design phase include the type of applications, the number and installed locations of reference devices, size of service area, and wireless device specifications (e.g., wireless technologies, radio frequency channels, and transmit power) $[5,8]$.

In the existing literature, on the system design phase, the research focused on various issues related to meeting required indoor positioning performance. In [9-11], the authors investigated the impact of reference node $(\mathrm{RN})$ placement on location accuracy performance. They compared the positioning performance obtained from different $\mathrm{RN}$ placements, such as comparing the placement of various basic geometric layouts [9], symmetrical/unsymmetrical layouts [10], or overlapping and hierarchical clustering [11]. Other system design researches aimed at developing the RN-placement techniques to determine the optimum number and location. These researches focused on essential issues that should be considered in the design of wireless indoor positioning systems, such as the radio signal coverage requirements $[12,13]$, the minimization of localization error [14], and the selection of the fitness values for the RN-placement techniques [15].

Second, in the localization phase, the physical information at the target location is measured and then analyzed to estimate its coordinates using localization algorithms. An example of these localization algorithms include the algorithms based on the Triangulation approach, such as Time Difference of Arrival (TDOA) [16] and Angle of Arrival (AOA) [17]. Another example of a localization approach called Scene Analysis utilizes a database of received signal strength (RSS) patterns recorded during the offline phase (i.e., location fingerprint database). The sample vectors of RSS values are matched with the patterns in the database during the online phase to estimate the location of the target $[4,18]$. The algorithms used in the Scene Analysis approach are the probabilistic methods [18], the Weighted K-Nearest Neighbor (WKNN) [19, 20], and the neural networks [21].

Based on the localization phase, several recent researches have aimed at investigating the properties of the RSS values inside the building. The findings of these analyses are needed in order to understand the underlying features of RSS characteristics, such as the distribution of RSS values [22] and the effect of the human body on RSS values [23]. Other researches have aimed at the development of the underlying mechanism for the localization algorithms, whereby those researches focused on improving the accuracy and the precision performance [24] and on reducing the computational complexity in location estimation [25].
One of the main challenges in wireless indoor positioning systems is the reliability. RN failures during the online (location determination) phase cause RSS values to be unavailable. This leads to performance degradation of localization accuracy and a lack of system reliability. In the design of indoor positioning systems, reliability is the capability required in order to maintain the functionality of the location determination in uncontrollable environments. In such cases, some RNs in the systems may fail, which could affect the entire operation of the indoor positioning system [26]. Under such unexpected situations, the performance accuracy of the system could drop by almost half [27]. According to the literature reviewed in Section 2, although some existing works have studied the reliable network design problem under node failure [28-30], they only investigated the provisioning of systems designed for telecommunications networks, and these network design solutions cannot be used effectively for indoor positioning systems. Furthermore, the system design for an indoor positioning system that supports the $\mathrm{RN}$-failure scenario during the online phase has not yet been investigated in the existing works. Thus, in this article, we will investigate how to design an RN-placement technique for robust indoor positioning systems that can overcome the problem of when some RNs fail. The major contributions of our article are as follows:

(i) We propose a robust system design for wireless indoor positioning systems based on the location fingerprinting technique. The proposed system design is provisioned to support the robust operation both during a normal situation and when some RNs fail. Our proposed design can be applied to various service area structures ranging from single-floor to multiple-floor environments.

(ii) We have developed a novel mathematical formulation using a Binary Integer Linear Programming (BILP) approach that employs simulated annealing (SA) based on the heuristic solution technique to solve the design problem for wireless indoor positioning systems.

The remainder of this manuscript is organized into six sections as follows. In Section 2, we briefly summarize existing works on the system design for indoor positioning systems. Section 3 provides the problem definition and the problem formulation. Section 4 describes the experimental environment, the wireless transceivers, and the setup parameters used in this work. Section 5 presents the experimental results and discussion. Finally, Section 6 concludes the findings of this article.

\section{Related Works}

In wireless sensor network (WSN) system design, the positioning of the nodes can affect numerous network performance metrics. The placement of any node will affect overall data collection and must take into account the condition of the physical environment. To ensure usability, it is necessary to propose a reasonable method for the installation of nodes in the network [31]. Moreover, many current applications 
require the wireless network to continue functioning under unexpected situations and hostile environments, such as node failures. For these reasons, the reliability of the network also requires considerable interest. Recently, several studies have focused on different system designs of reliable and survivable wireless networks to support robust operation in uncontrollable environments. These studies have proposed system design methods that can achieve the reliability and survivability requirements for wireless networks.

In the system design of telecommunications networks, node failures will result in the complete outage of some wireless links in the network. This situation can cause disruption to the network's connectivity and loss of availability. Therefore, several studies have focused on the survivability of the network systems and how to handle the problem of failure situations. Correia et al. [28] presented a fault-tolerance network plan for the wireless optical broadband access network (WOBAN). Their fault-tolerance model can be applied to any scenario of optical wireless failure. Liu et al. [29] presented a reliable mixed-integer programming model for the IP layer network. Their system design model was provisioned to support faulty relay nodes caused by hardware failures or by an overloaded network. Luo et al. [30] presented a gateway placement approach for wireless mesh networks (WMNs). The optimal number and locations of the gateways are provisioned to reduce the gateway interference and provide fault-tolerance assurance. Based on the system design solutions presented in the literature, node failures in a telecommunications network may cause the network either to become disconnected or to have unavailable wireless links in the network. In particular, this may result in complete network outage. To address this problem, several research studies have proposed reliable system designs, whereby those developed systems could still function after some failure of certain network components, usually based on the provision of backup links or bandwidth management schemes.

While the worst-case scenario of node failures in a telecommunications network may result in the network's unavailability, the situations are different for indoor positioning systems. The faulty RNs in indoor positioning systems may decrease the location performance accuracy, although the systems can still provide location estimations. However, the localization accuracy performance of such systems may be less than half of normal if the system is affected by RN failures during the online estimation phase. There is a significant difference between the telecommunications systems and the indoor positioning systems in that the general network design solutions cannot be used effectively for indoor positioning systems.

With regard to the system design for indoor positioning systems, several studies have focused on addressing various design issues in order to meet performance requirements. In $[12,32,33]$, the authors proposed a system design for indoor positioning systems based on location fingerprinting techniques. Zhang et al. [12] presented a mathematical formulation of $\mathrm{RN}$ placement that aims to minimize the number of RNs, while ensuring that their locations still have sufficient coverage of the service area. Sharma et al. [32] proposed an RN-placement method that aims to minimize the total number of similar fingerprints so as to achieve better location accuracy. Fang et al. [33] presented a framework for linking the RN placement and the positioning performance. The objective of their algorithm was to choose a suitable set of $\mathrm{RN}$ locations so that the signal-to-noise ratio (SNR) was maximized.

Other research works on system design focused on improving the indoor positioning systems based on a triangulation approach [13-15]. Aomumpai et al. [13] proposed a system design approach based on a genetic algorithm to find the optimal solution of RN placement in an RSS-based localization approach. They focused on minimizing the average localization error and maximizing the signal coverage of the service area. Redondi et al. [14] presented an $\mathrm{RN}$-placement method for indoor positioning systems based on the Cramer-Rao lower bound (CRLB) approach. The objective of their system design was to minimize localization errors when operating with a limited number of RNs due to budget constraints are fixed. Merkel et al. [15] presented an optimal $\mathrm{RN}$-placement approach for an indoor positioning system based on distributed range-free localization. They focused on achieving the optimal coverage of a certain area while simultaneously minimizing the necessary number of RNs.

In the literature, existing system designs for indoor positioning systems limit their focuses to the achievement of location accuracy or the provision of signal radio coverage in the service area. Furthermore, the major design concern in the reliability of indoor positioning systems under the faulty RNs during the online phase has not yet been investigated in the exiting works. Unfortunately, existing solutions to survivable network design problems for telecommunications networks cannot be used effectively for indoor positioning systems. Based on these knowledge gaps, the design of RN placement for robust indoor positioning systems is still an open research issue. Therefore, in this work, we propose a robust system design model for wireless indoor positioning systems based on location fingerprinting techniques. Our proposed model aims to place a suitable number of RNs and to determine their locations whereby their placement is provisioned to support robust system operation both during a normal situation and when some RNs have failed.

\section{Problem Definition and Formulation}

In this section, we describe our mathematical models for the problem of RN placement in indoor multifloor positioning systems. First, in Section 3.1, the problem definition of wireless indoor multifloor positioning systems under RNfailure scenarios is described. Then, in Section 3.2, we explain our mathematical formulation for the robust system design problem. Finally, in Section 3.3, the overall framework of the solution technique is described.

\subsection{Definition of System Design for Wireless Indoor Positioning} Systems. In wireless indoor positioning system designs, placement of insufficient RNs can lead to accuracy performance degradation. Furthermore, the system may achieve less than half of its intended performance levels during the 
online estimation phase in the event of RN failures [27]. Under unexpected situations, such as some RNs being faulty during the online phase, unreliable location results are presented because of the unavailability of RSS component(s) during the location estimation process. For example, Figure 1 illustrates an example of the structure of an indoor positioning system in a three-story building (e.g., a smart office building) under the $\mathrm{RN}$-failure scenario in which two RNs have failed. In this diagram, four RNs are installed on each floor. The dashed lines represent the RSS values that a target node receives from all RNs. In Figure 2, one $\mathrm{RN}$ on the 1st floor and another $\mathrm{RN}$ on the 2 nd floor have become unavailable. This may be caused by hardware failures or software errors. In either case, the online scanned RSS values of the target node would not have signals from these RNs and the faulty RNs could reduce the location estimation performance.

To ensure the reliability of indoor positioning systems, a system design model that can handle situations of signal unavailability due to $\mathrm{RN}$ failure is required. Furthermore, the $\mathrm{RN}$-placement design model as a major system concern in the reliability of indoor positioning systems in the event of some faulty RNs during the online phase has not been investigated.

3.2. Robust System Design Model. In this section, we describe the robust system design for wireless indoor positioning systems. The proposed system design is called the RobustMaximum Summation of Max RSSI (R-MSMR), which is enhanced from our previous mathematical model presented in [34]. The task of the proposed R-MSMR is to place a sufficient number of RNs and determine their optimum locations, which may be located on a single floor or on multiple floors. In designing such a system, we focus on the network planning in terms of the wireless network infrastructure in which RNs serve as the wireless nodes in the network. The number of $\mathrm{RNs}$ and their locations are determined to ensure that the radio signal coverage is sufficient for the service area. In particular, the robust system design should result in a design solution that supports robust operation both during a normal situation and when some RNs have failed. We formulate the mathematical formulation of the robust system design problem as a Binary Integer Linear Programming (BILP) problem. This deals with models that are the same as linear programming with one additional restriction. The variables of BILP have integer values in which all variables are binary variables [35]. We adopt Simulated Annealing (SA) based on the heuristic solution technique to solve the BILP problem for the R-MSMR because of its simplicity and effectiveness. The SA is a variation of a hill-climber heuristic search approach. The SA allows the search to move to nonimproving solutions with a certain probability. This allows the SA search to avoid being trapped at local optima [36]. The R-MSMR based on BILP consists of two main components: the objective function and the constraints. The notations defined and used in the mathematical formulation of the robust system design problem are summarized in Table 1.
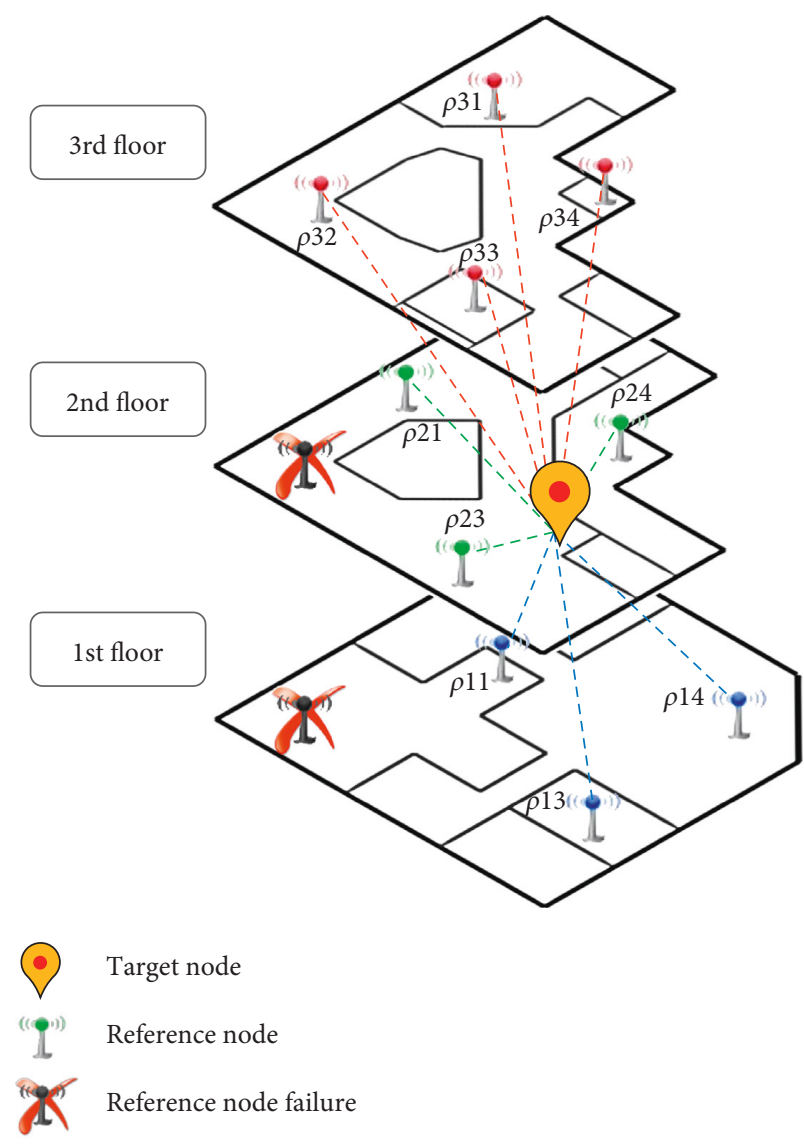

Figure 1: Floor determination schematic diagram.

We developed the robust system design in order to solve the $\mathrm{RN}$-failure problem in wireless indoor positioning systems. The proposed system design not only achieves high positioning accuracy during normal situations but also yields reliable location results under unexpected situations such as RN failures. The main objective of the proposed R-MSMR is to place a sufficient number of RNs in optimum locations so that the system can achieve a maximizing summation of the maximum RSS at the signal test points (STPs) received from the RNs installed in the service area as written in the objective function:

$$
\text { Maximize } \sum_{\forall i \in T} \max _{\forall j \in B}\left(S_{i j} P_{i j}\right) \text {. }
$$

The constraints for the R-MSMR are as follows: constraint (2) states that STP $i$ is under the coverage of $\mathrm{RN} j$ if the signal strength received at STP $i$ from $\mathrm{RN} j\left(P_{i j}\right)$ is greater than the sensitivity threshold $P_{T}$. This constraint is used to ensure a high quality of radio signal propagation in the service area. Constraint (3) enforces that each STP must be able to receive a signal from at least a recommended number of RNs for a given accuracy and reliability which are specified with a summation of the accuracy index $\alpha$ and reliability index $R$. The accuracy index $\alpha$ is a minimum number of radio signals obtained from RNs that should provide a good performance according to Kaemarungsi [8]. A large value (more than the recommended value) will result in higher accuracy and precision while a small value will decrease 


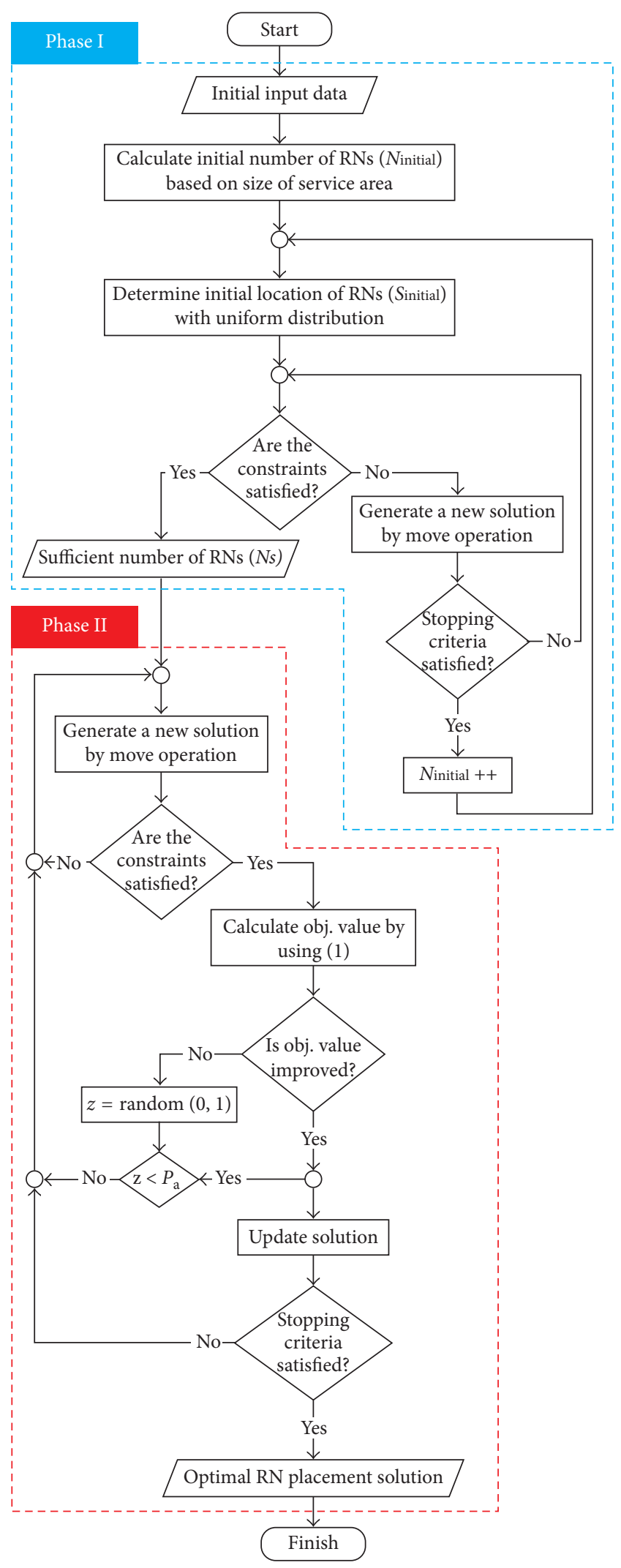

FIgURE 2: Framework of the solution technique.

performance of both accuracy and precision. However, a large value of the accuracy index $\alpha$ will also require a high cost of node infrastructure installation. The reliability
TABLE 1: Notations.

\begin{tabular}{|c|c|}
\hline \multicolumn{2}{|c|}{ Sets } \\
\hline$B$ & $\begin{array}{c}\text { A set of candidate sites (CS) that are locations for } \\
\text { installing the reference node (RNs) }\end{array}$ \\
\hline$T$ & $\begin{array}{c}\text { A set of signal test points (STP) that are locations for } \\
\text { testing received signal strength }\end{array}$ \\
\hline \multicolumn{2}{|c|}{ Decision variables } \\
\hline$c_{j}$ & $\begin{array}{l}\text { A binary }\{0,1\} \text { variable that equals } 1 \text { if the } \mathrm{RN} \text { is } \\
\text { installed at site } j, j \in B ; 0 \text { otherwise }\end{array}$ \\
\hline$S_{i j}$ & $\begin{array}{c}\text { A binary }\{0,1\} \text { variable that equals } 1 \text { if the STP } i \text { is } \\
\text { assigned to RN } j, i \in T \text { and } j \in B \text {; } 0 \text { otherwise }\end{array}$ \\
\hline \multicolumn{2}{|c|}{ Constant parameters } \\
\hline $\begin{array}{l}P_{i j} \\
P_{T}\end{array}$ & $\begin{array}{c}\text { The RSS that STP } i \text { receives from } \mathrm{RN} j, i \in T \text { and } j \in B \\
\text { The RSS sensitivity threshold }\end{array}$ \\
\hline$N_{s}$ & $\begin{array}{c}\text { The sufficient number of RNs installed in the service } \\
\text { area }\end{array}$ \\
\hline$\alpha$ & $\begin{array}{l}\text { The accuracy index or the recommended number of } \\
\text { RNs that should provide higher accuracy and } \\
\text { precision according to [8] }\end{array}$ \\
\hline$R$ & $\begin{array}{l}\text { The reliability index or the number of RNs that } \\
\text { provide reliability performance }\end{array}$ \\
\hline
\end{tabular}

index $R$ is a margin of the number of RNs that is acceptable to fail. Constraint (4) enforces that there is a sufficient number of RNs $\left(N_{S}\right)$ to be utilized in the service area. Constraint (5) specifies that STP $i$ can receive a signal from $\mathrm{RN} j$ if $\mathrm{RN} j$ is installed.

$$
\begin{aligned}
S_{i j}\left(P_{i j}-P_{T}\right) & \geq 0, \quad \forall i \in T, \forall j \in B, \\
\sum_{\forall j \in B} S_{i j} \geq \alpha+R, \quad \forall i \in T, & \\
\sum_{\forall j \in B} c_{j} & =N_{S}, \\
S_{i j} & \leq c_{j}, \quad \forall i \in T, \forall j \in B .
\end{aligned}
$$

3.3. Solution Technique for R-MSMR. In this section, we describe the overall framework of the solution technique. Figure 2 shows the framework of the solution technique for the R-MSMR. Initial input data of the solution technique are divided into two inputs. The first input involves the physical properties of the service area (i.e., the floor dimension and the total number of floors) and the wireless transceiver information (i.e., the $\mathrm{RN}$-signal coverage area). The second input specifies the parameters for fundamental calculations of the solution technique, including a set of signal test points (STPs), a set of candidate sites for installing RNs (CS), the RSS data of all STPs that have been received from RNs in Watts $\left(Z_{R S S}\right)$, the accuracy index, and the reliability index. The output from the solution technique is the optimal number of RNs installed and their locations that ensure the continued effectiveness of the system when some RNs in the system fail. Note that this work considers the discrete candidate sites for installing RNs in order to reduce the computational complexity of the system design. 
In the proposed framework, the solution technique consists of two phases. Phase I aims to generate a good starting solution that provides a sufficient number of RNs installed $\left(N_{S}\right)$. First, an initial number of RNs $\left(N_{\text {initial }}\right)$ as a minimum starting number of RNs installed is calculated based on the size of the service area. The starting number is then used to determine the initial location of the RNs $\left(S_{\text {initial }}\right)$, whereby the RNs are uniformly distributed across the service area. Then, the process is repeated until the set of optimization constraints (2)-(5) are satisfied. In each iteration, move operation based on the SA approach is used to generate the new possible solution (called the neighbor solution), in which specific attributes of the current solution are adjusted [36]. After that, a solution that provides a sufficient number of RNs is obtained.

In Phase II, the SA approach based on the heuristic solution technique is used to determine the optimal location of the RNs $\left(S_{\mathrm{o}}\right)$ which is specified with the number of RNs obtained from Phase I. In this phase, the cost of each RN-placement solution is calculated by using the evaluation function as written in (1). Unlike a general heuristic solution technique which only moves to a solution that improves the objective function, SA allows the search to move to nonimproving solutions with the probability of accepting. The acceptance probability for the optimization problem is written as (6), where $\Delta$ cost is the difference between the cost of a neighbor solution and the cost of the current solution. The control temperature $\tau$ is used to control search progresses, in which the temperature is reduced according to the increasing number of iterations in the annealing schedule. When a worse solution is met, a random number $z$ in the ranges $[0,1]$ is generated and is compared to the probability of accepting. The worse solution is rejected when a random number is lower than the acceptance probability value $\left(z<P_{\mathrm{a}}\right)[36]$ :

$$
P_{\mathrm{a}}=e^{-(\Delta \cos \mathrm{t} / \tau)}
$$

Then, the SA process continues until a stopping condition is reached. Finally, the solution that provides the optimal RN placement for indoor positioning systems is obtained. In particular, this optimal RN-placement solution can support the robust operation either during a normal situation or when there is a failure of some RNs. Figure 3 illustrates an example of the RN-placement solution obtained from the proposed R-MSMR, in which the reliability index is equal to two.

\section{Experimental Setups}

To evaluate the R-MSMR results, the positioning performance of the R-MSMR was analyzed, and the results were compared with other RN-placement designs under a normal situation and when some RNs have failed. In particular, the RN-placement approaches were compared between the proposed R-MSMR and the following three different designs: the coverage and uniform placement (Uniform) model, the Maximize-Sum of Maximum RSS (MSMR) model [34], and Phase I (i.e., sufficient number of RNs obtained from the proposed design) with the uniform placement (PhI-Uni). For example, the objective of the Uniform is to place the RNs in a service area, in which the whole service area must be able to receive signals from at least one $\mathrm{RN}$. In order to estimate the target location inside the multi-story buildings, the RMoS floor algorithm [37] and the active Euclidean distance technique based on the location fingerprinting approach are used. Instead of matching the RSS patterns obtained from all the RNs in the service area as occurs with the traditional Euclidean distance technique, the active Euclidean distance technique only considers the RSS values that are transmitted from the available RNs (i.e., active RNs) for a matching RSS pattern process. The RMoS floor algorithm is used to determine the floor number of the target, while the active Euclidean distance is used to estimate the target location in coordinates $(x, y)$, in which the matching process of the algorithm considers only the available RSS received from any $\mathrm{RN}$ installed. The core of this study can be divided into two objectives:

(1) To compare the positioning performance of indoor positioning systems based on the location fingerprinting technique in which the system is employed by different system designs (which will be discussed in Section 5.2).

(2) To analyze the impact of different RN-failure patterns on the accuracy of indoor multifloor positioning systems (which will be discussed in Section 5.3).

4.1. Experimental Settings. Figure 4 illustrates the floor layouts of a three-story building, which consists of office zones, classrooms, student lounge, and common rooms. The dimension of each floor is approximately $75 \mathrm{~m}$ (width) $\times$ $75 \mathrm{~m}$ (length). We divided the system design experiment of the service area into three scenarios. Scenario 1 considers a single-floor service area in which the service area is located on the first floor. Scenario 2 considers a two-floor service area that covers both the first and the second floor of the building. Scenario 3 considers a three-floor service area. Note that each floor has open space in the center. In each scenario, two parameter sets with the same configuration are used, consisting of the signal test points and the candidate sites for the RN locations as shown in Table 2 .

We assign the grid spacing of the fingerprint locations at four meters as shown by the blue cross in Figure 4. A total number of 158, 316, and 474 test points (i.e., target locations) were randomly selected for all three test scenarios, respectively. Note that these numbers of test points were obtained by determining the sample size with confidence intervals [38]. Table 2 shows a summary of the parameters used in our experiments. We conducted four value tests of the reliability index $R$ (i.e., $R=1,2,3$, and 4) to observe how it impacts indoor positioning performance. Note that we define the input parameters of the R-MSMR on SA terms with the cooling ratio $\varphi=0.9$, the stopping temperature $\tau_{\text {stop }}=0.01$, and the maximum count of the no-improvement iteration $n_{\text {worse_max }}=2000$. These constraints follow the recommendations of Kirkpatrick et al. [36]. 

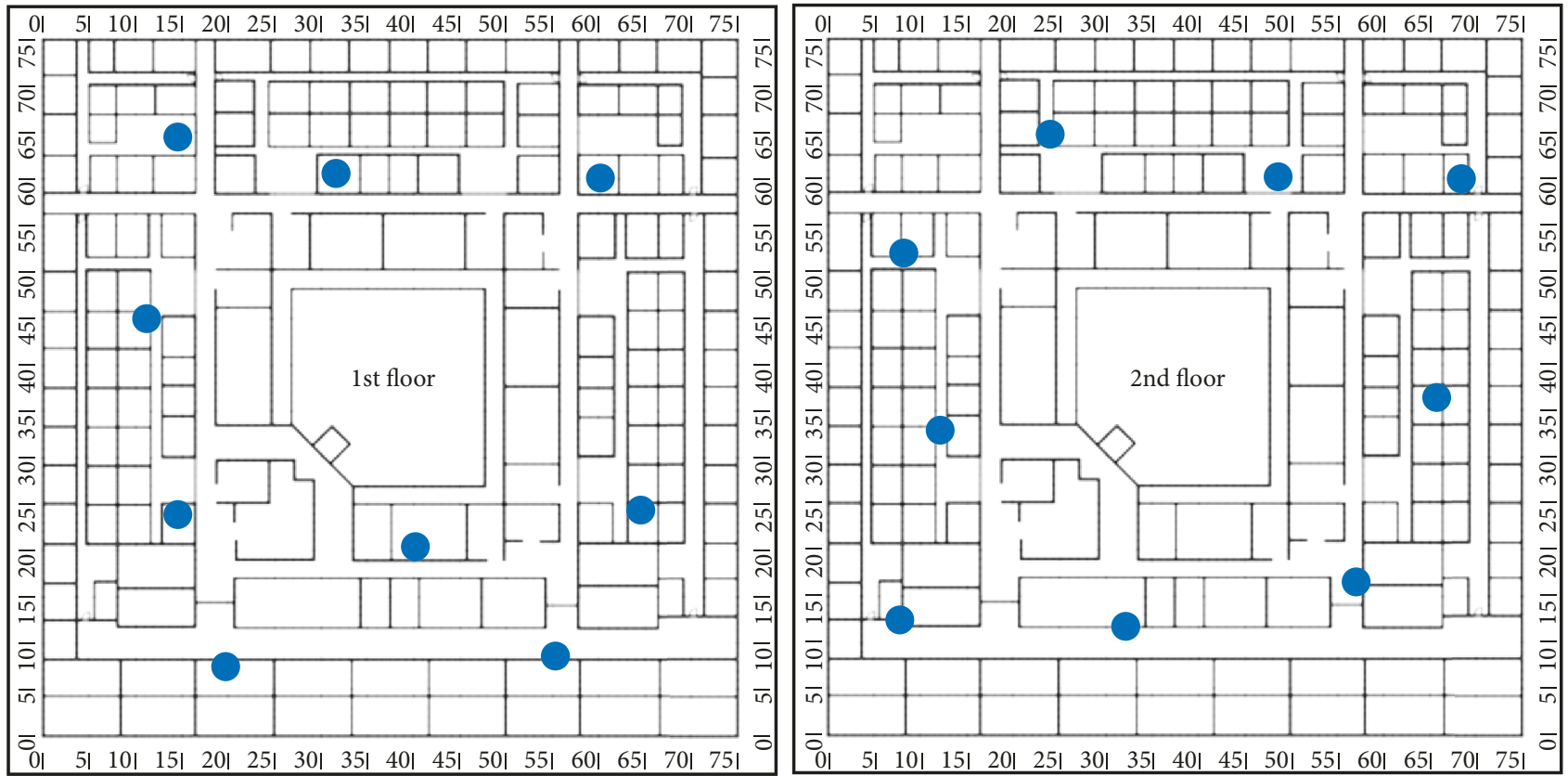

FIgURE 3: Example of the R-MSMR with $R=2$ for the two-story building.

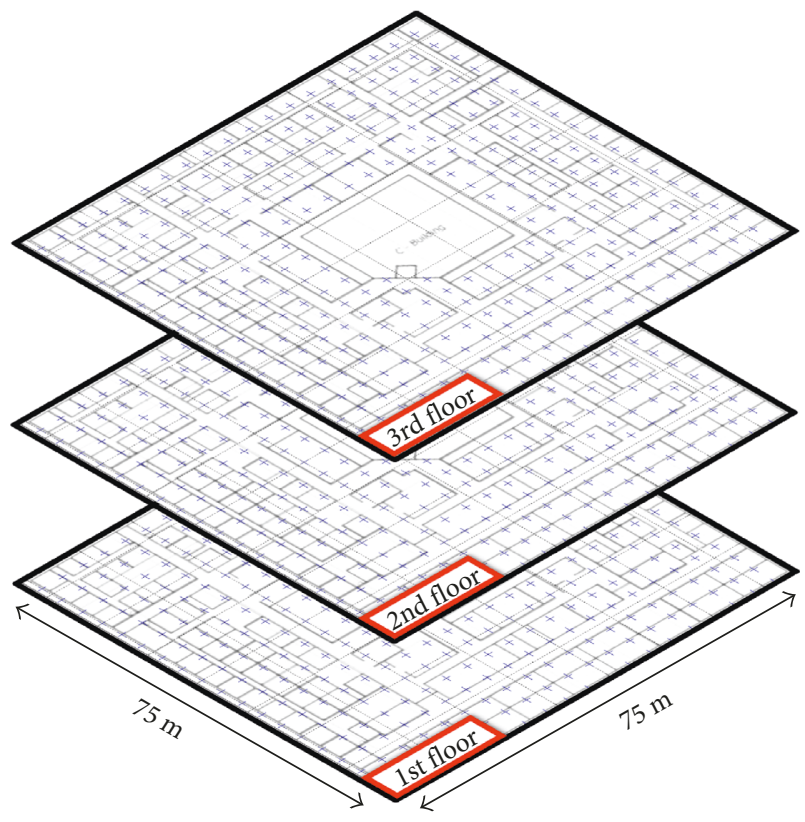

FIgURE 4: The three-story structure with the assigned STPs location.

4.2. Experimental Equipment. Figure 5 illustrates the experimental equipment installed on each floor. This includes RNs which are placed at a height of two meters. The target node is connected to a computer notebook on which the localization technique is executed. The height of the target node is 0.8 meters. IEEE 802.15.4 wireless transceivers were deployed in this work. Each device (RNs and target) has a Freescale MC13224V third-generation chipset with builtin ARM7TDMI processors. The antennas of the wireless transceivers are the inverted F-shape antennas and SMA antennas [39]. All devices are operated at $2.480 \mathrm{GHz}$ (i.e., channel 26 of IEEE 802.15 .4 standard). This is to avoid or minimize the interference from $\mathrm{Wi}-\mathrm{Fi}$ networks in the area. The transmit power is $+3 \mathrm{dBm}$, while the typical sensitivity of the wireless transceivers is $-95 \mathrm{dBm}$. A typical range (indoors, nonline of sight) is about 30 meters. The target node will gather the RSS values that are transmitted from the RNs in the service area. These measured RSS values are used to estimate the location of the target node. Note that the proposed solution technique calculates the RSS data in linear scale (i.e., Watts). Therefore, we did not use $\mathrm{dBm}$ in our calculation because it has limitations in terms of the multiplication and division of log scales. The sampling rate of wireless transceivers in this work is 1 sample for every 3 seconds. Note that we only consider the stationary node in our experimental study. Table 3 summarizes the specifications of the wireless transceivers used in our experiments.

\section{Results and Discussion}

In this section, several aspects of the numerical results of the RN placement are discussed. First, in Section 5.1, the numerical results of different $\mathrm{RN}$-placement designs for indoor positioning systems are described. Next, in Section 5.2, we provide a comparative performance evaluation of the R-MSMR versus other system designs. Finally, in Section 5.3, the impact of RN-failure patterns on the accuracy performance of indoor positioning systems is analyzed.

5.1. System Design Results. Figures 6-8 illustrate the results of the RN-placement designs for Scenarios 1 to 3 , respectively. Each graphic reports the number and the locations of the RNs obtained from four different system designs, which are the Uniform, the MSMR, the R-MSMR with $R=2$, 
TABLE 2: Summary of parameters used in the experiments.

\begin{tabular}{lc}
\hline Parameter & Details \\
\hline $\begin{array}{l}\text { Floor dimensions } \\
\text { RNs placement } \\
\text { Number of signal test points }(\mathrm{STP}) \text { and candidate } \\
\text { sites }(\mathrm{CS})\end{array}$ & $75 \mathrm{~m} \times 75 \mathrm{~m}$ (for 1st, 2nd, and 3rd floor) \\
Grid spacing of fingerprint locations & Uniform, MSMR, R-MSMR, and PhI-Uni \\
Number of test points (i.e., target locations) & Scenario 2, and 984 locations for Scenario 3 \\
The RSS sensitivity threshold $\left(P_{\mathrm{T}}\right)$ & $4 \mathrm{~m} \times 4 \mathrm{~m}$ \\
Accuracy index $(\alpha)$ & 158 locations for Scenario 1,316 locations for Scenario 2, \\
Reliability index $(R)$ & and 474 locations for Scenario 3 \\
Floor determination technique & $0.1 \mathrm{pW} \mathrm{(i.e.,}-100 \mathrm{dBm}$ ) \\
Localization technique & 4 [8] \\
\hline
\end{tabular}

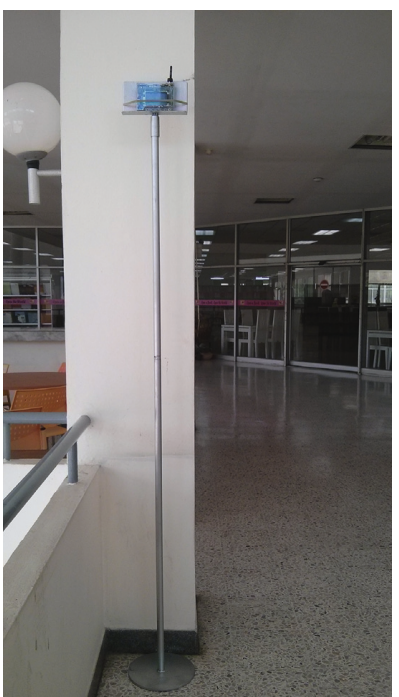

(a)

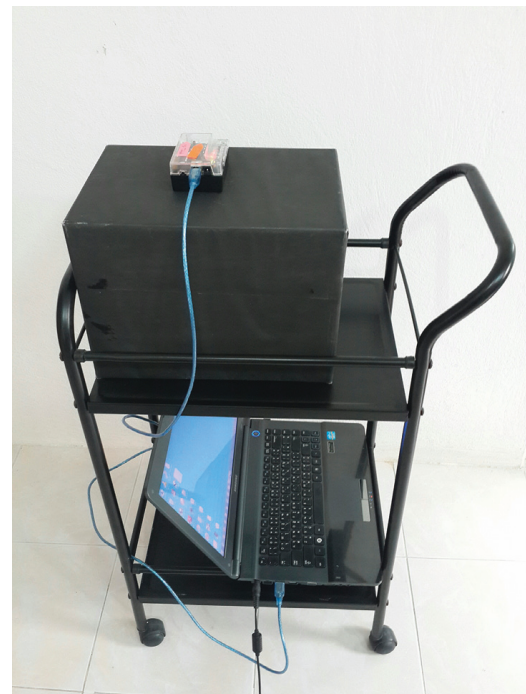

(b)

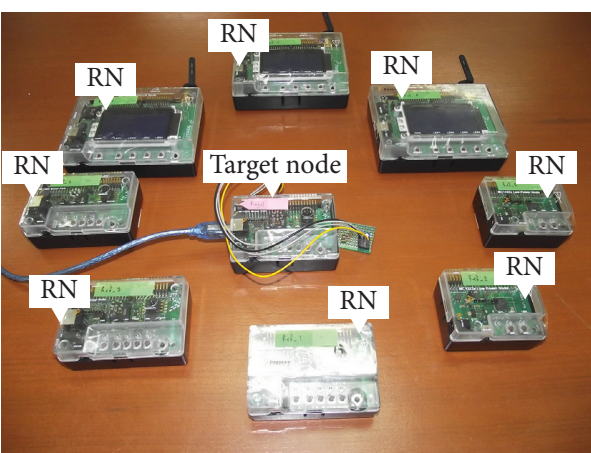

(c)

FIGURE 5: Experimental equipment used in this work. (a) Reference node (RN). (b) Target node on a cart with laptop PC. (c) Experimental equipment used in this work.

TABLE 3: Specifications of the wireless transceivers.

\begin{tabular}{lc}
\hline Specification & Details \\
\hline Manufacturer & Freescale \\
Chipset & $\mathrm{MC} 13224 \mathrm{~V}$ \\
Frequency range & $2.405 \mathrm{GHz}$ to $2.480 \mathrm{GHz}$ \\
Operating channel & $\mathrm{CH} 26(2.480 \mathrm{GHz})$ \\
Rx sensitivity & $-95 \mathrm{dBm}$ \\
Transmit power & $+3 \mathrm{dBm}$
\end{tabular}

Antenna

Inverted F-antenna and SMA antenna

and the PhI-Uni. In these results, we found that the Uniform has the lowest number of RNs installed in all three scenarios, while the R-MSMR with $R=2$ has the highest number of $\mathrm{RNs}$ installed for all three scenarios. The reason for the $\mathrm{R}-\mathrm{MSMR}$ requesting a higher number of $\mathrm{RN}$ s to be installed than other system designs is that the proposed R-MSMR structure is designed to provide high positioning accuracy during a normal situation and also achieve reliable location results when some RNs in the system fail. Therefore, our algorithm seeks to distribute the $\mathrm{RN}$ locations across the service area while maintaining high quality radio signal coverage.

5.2. Performance Evaluation of the System Designs. In this section, we evaluate the location estimation performance of different system designs. In particular, the proposed R-MSMR was compared with the Uniform, the MSMR, and the PhI-Uni under the same experimental settings as shown in Table 2. The RMoS floor algorithm and the active Euclidean distance technique based on the location fingerprinting approach were used to estimate the target locations in coordinates $x, y$, and floor. Two different RN scenarios were considered in our experiments. The first scenario is a fault-free scenario in which all RNs worked properly. The second scenario is a $3 R N$-failure per floor scenario in which three of the RNs on each floor fail. The experimental results of the 3RN-failure per floor were averaged from four faulty patterns. For example, we randomly selected and turned off 


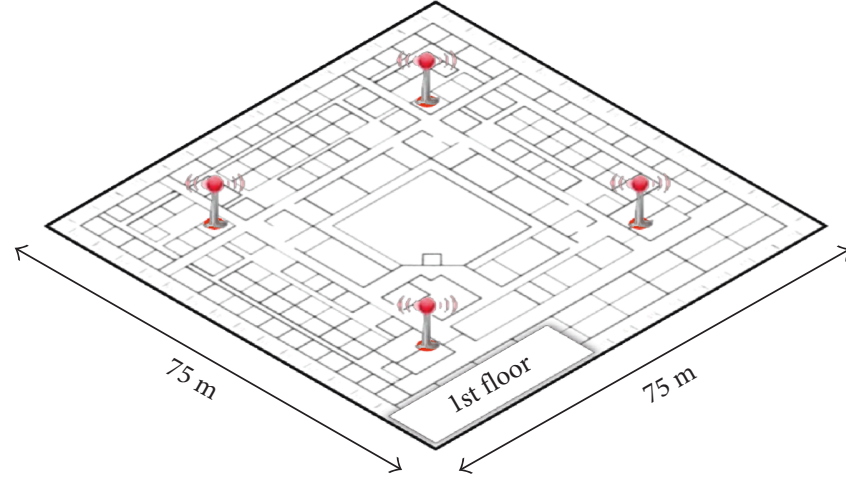

(a)

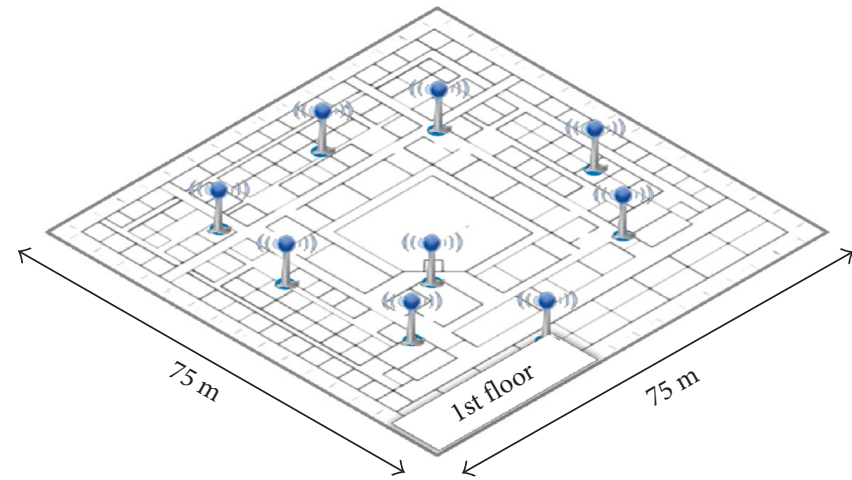

(c)

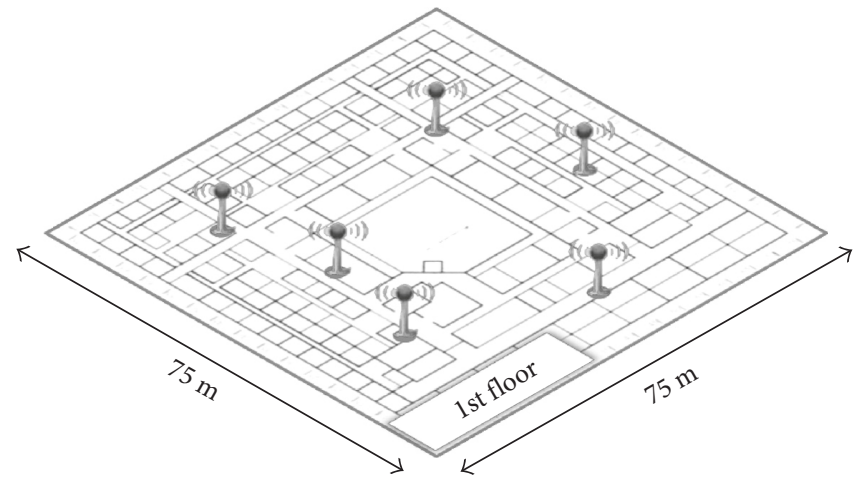

(b)

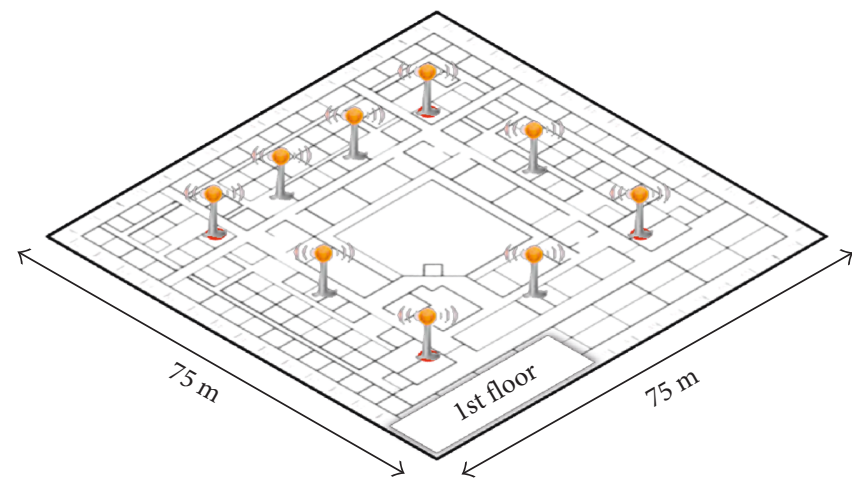

(d)

Figure 6: The RN placement designed for Scenario 1. (a) Uniform (4 nodes). (b) MSMR (6 nodes). (c) R-MSMR, $R=2$ (9 nodes). (d) PhIUni (9 nodes).

three RNs on each floor to create four patterns of RN failure. Then, an average accuracy performance was computed from those four patterns of RN failure.

The positioning performance results of different system designs were divided into two sections. In Section 5.2.1, the results of the correct floor determination are presented. Then, in Section 5.2.2, the accuracy and the precision of four different indoor positioning systems that employ different system designs are compared.

5.2.1. Correct Floor Determination Results. Under the faultfree scenario, all four indoor multifloor positioning systems that employ different design structures were able to achieve results of $100 \%$ correct floor determination in both Scenario 2 and Scenario 3 (i.e., inside the two- and three-floor service area, resp.). Unlike the fault-free scenario, indoor multifloor positioning systems under the RN-failure scenario encountered problems from the missing RSS values during the online phase due to the three faulty RNs on each floor. Figure 9 compares the floor determination accuracy under the $3 \mathrm{RN}$ failure per floor scenario. The red bins, gray bins, blue bins, and yellow bins represent the correct floor determination percentages for the Uniform, the MSMR, the R-MSMR with $R=2$, and the PhI-Uni, respectively. From these results, we found that the floor determination accuracy of indoor multifloor positioning systems that employ the Uniform, the MSMR, and the PhI-Uni decreased in the event of faulty RNs.
In the results of Scenario 2 and Scenario 3, the Uniform had the lowest correct floor determination percentage of about $92.01 \%$ and $62.34 \%$, respectively. Similar floor determination results are seen in the MSMR and the PhI-Uni. In particular, we found that the insufficient RN placement had a significant effect on floor determination performance. The floor determination of the PhI-Uni which used a sufficient number of RNs $\left(N_{S}\right)$ had an incorrect floor number. The PhI-Uni did not provide $100 \%$ correct floor determination in either Scenario 2 or Scenario 3 (98.25\% and 91\%, resp.). These figures are different from the proposed system design, in which the R-MSMR with $R=2$ was able to achieve the highest correct floor determination percentage of up to $100 \%$ in both Scenario 2 and Scenario 3. Moreover, we found that the floor determination accuracy of the Uniform, the MSMR, and the PhIUni depended on the number of RNs which failed inside the building; the floor determination accuracy decreased in line with the rise in the number of $\mathrm{RN}$ failures.

This observation suggests that a larger number of the $\mathrm{RN}$ failures (i.e., considering six and nine $\mathrm{RN}$ failures as large numbers in Scenario 2 and Scenario 3, resp.) degrade the floor determination accuracy of the Uniform, the MSMR, and the PhI-Uni. Only the performance of the proposed R-MSMR was not affected by the increasing number of RN failures. It succeeded $100 \%$ in correct floor determination in the scenarios of both six and nine $\mathrm{RN}$ failures in the building. 


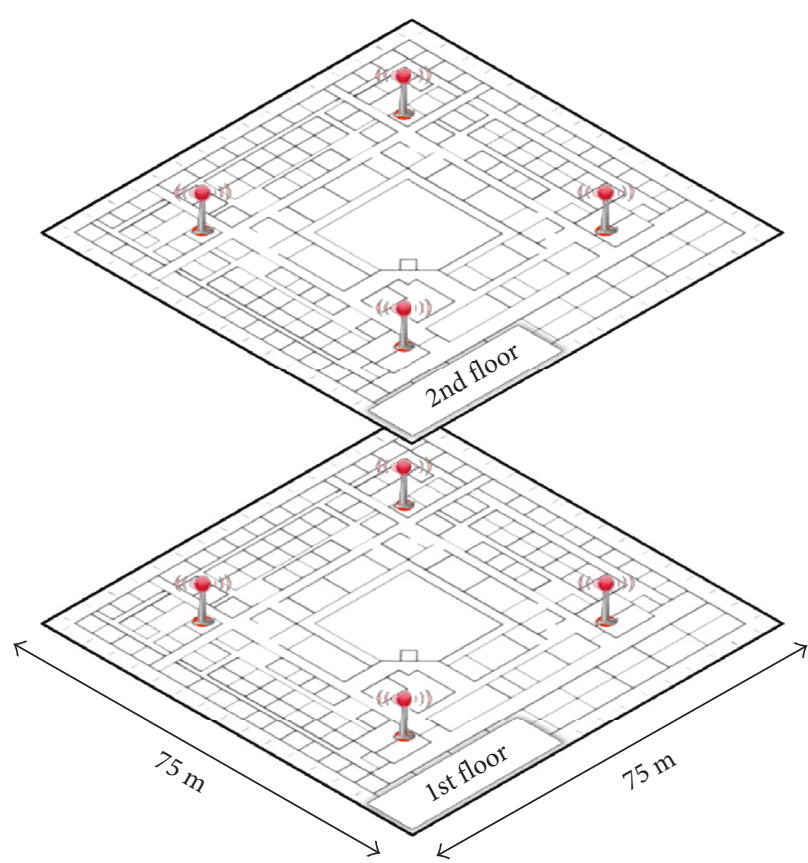

(a)

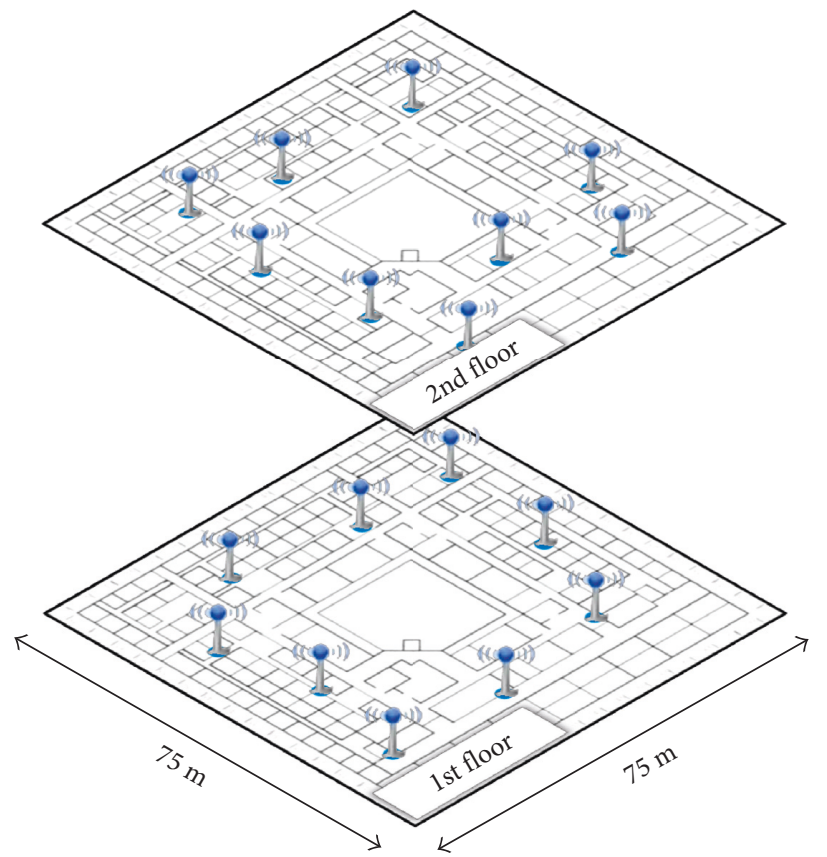

(c)

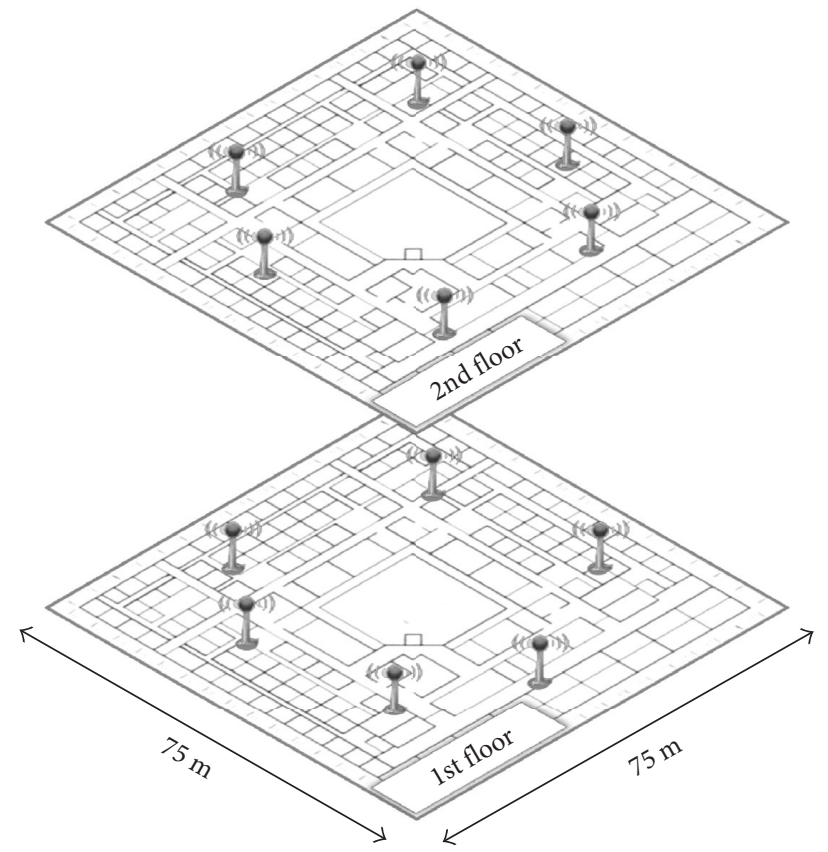

(b)

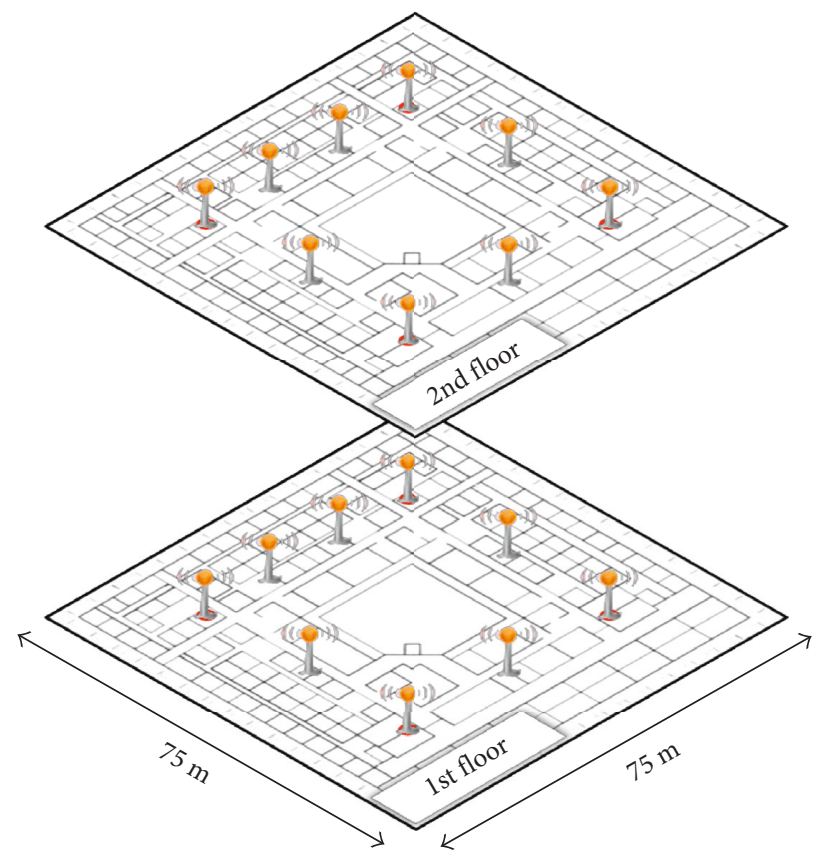

(d)

Figure 7: The RN placement designed for Scenario 2. (a) Uniform (8 nodes). (b) MSMR (12 nodes). (c) R-MSMR, $R=2$ (18 nodes). (d) PhIUni (18 nodes).

5.2.2. Results of the Positioning Accuracy $(x, y)$. Figures 10-12 report average error distances of indoor positioning systems that employ four different design structures for Scenario 1 to Scenario 3, respectively. We first consider the accuracy performance of the indoor positioning systems under the fault-free scenario as represented by empty bins. The proposed R-MSMR with $R=2$ shows a better accuracy performance than other system designs in all three scenario areas. For example, Figure 11 illustrates four different positioning results under the fault-free scenario in the twostory building of Scenario 2. The average error distance of the R-MSMR is 4.42 meters, while the average error distances of the Uniform, the MSMR, and the PhI-Uni are 6.35 meters, 5.20 meters, and 5.13 meters, respectively. R-MSMR has $30.4 \%, 15.0 \%$, and $13.8 \%$ better performance than the Uniform, the MSMR, and the PhI-Uni, respectively. In comparing the accuracy performance of all three service areas under the fault-free scenario, the proposed R-MSMR 


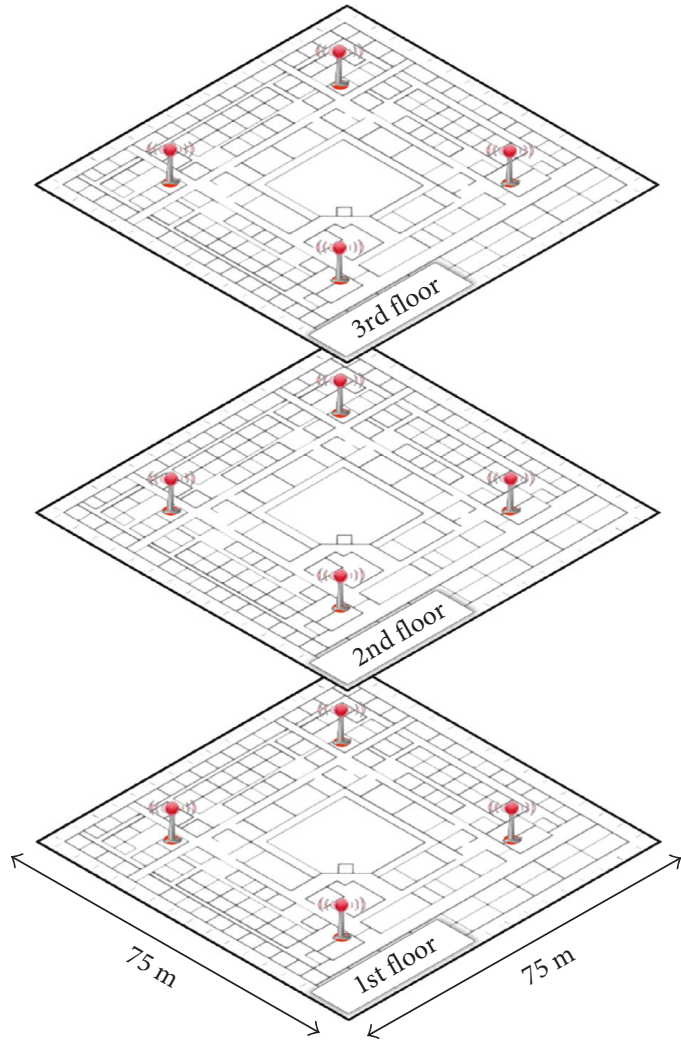

(a)

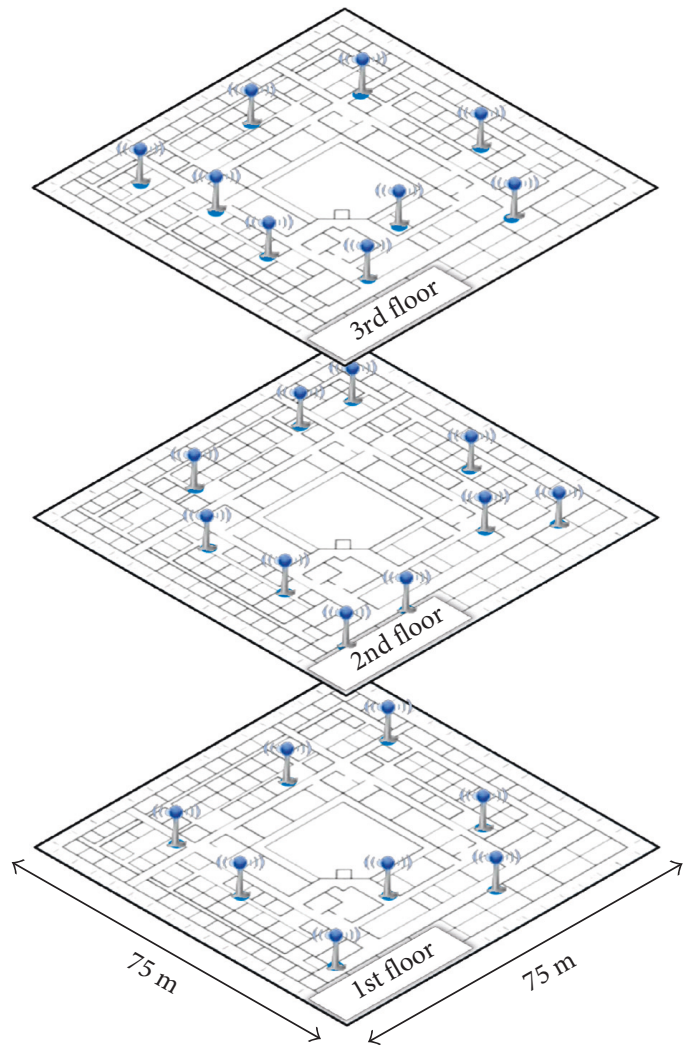

(c)

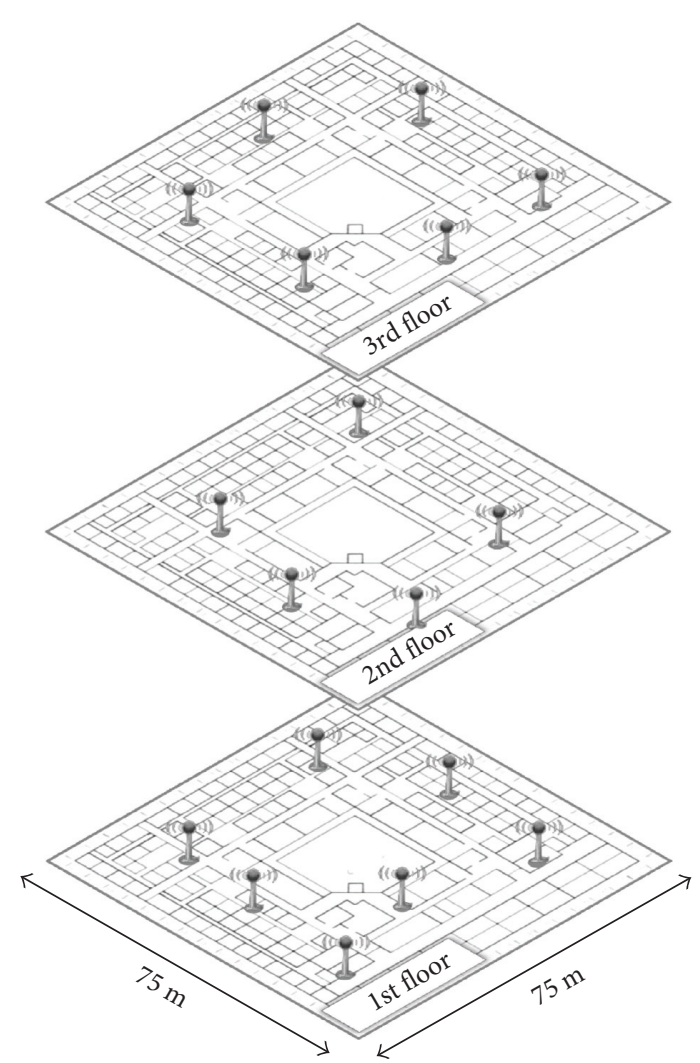

(b)

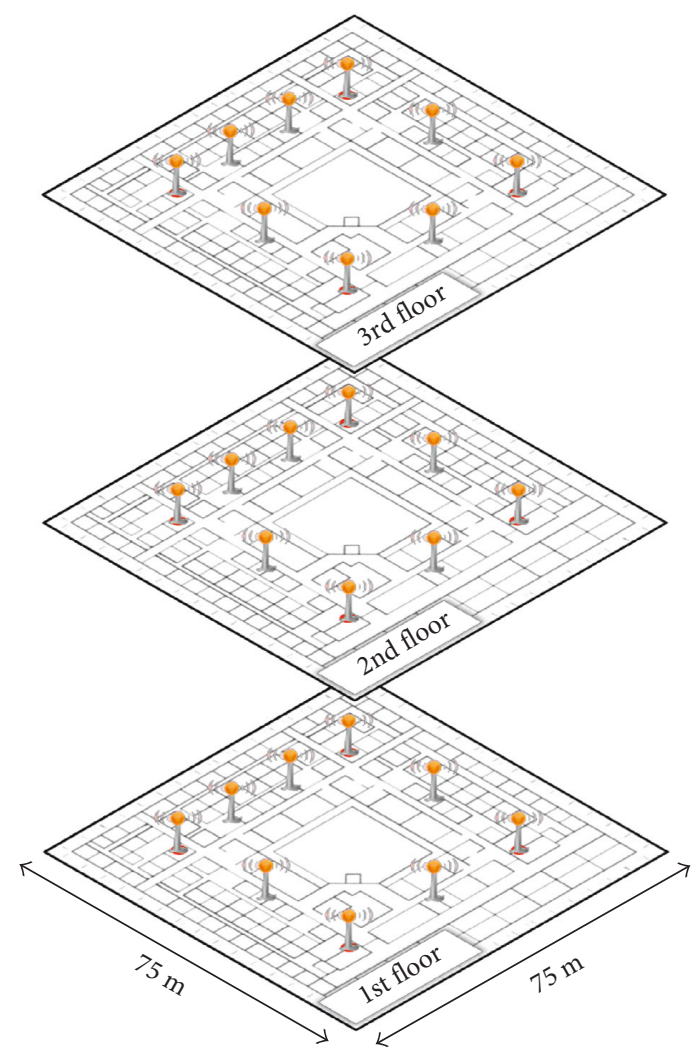

(d)

Figure 8: The RN placement designed for Scenario 3. (a) Uniform (12 nodes). (b) MSMR (18 nodes). (c) R-MSMR, $R=2$ (27 nodes). (d) PhIUni (27 nodes). 


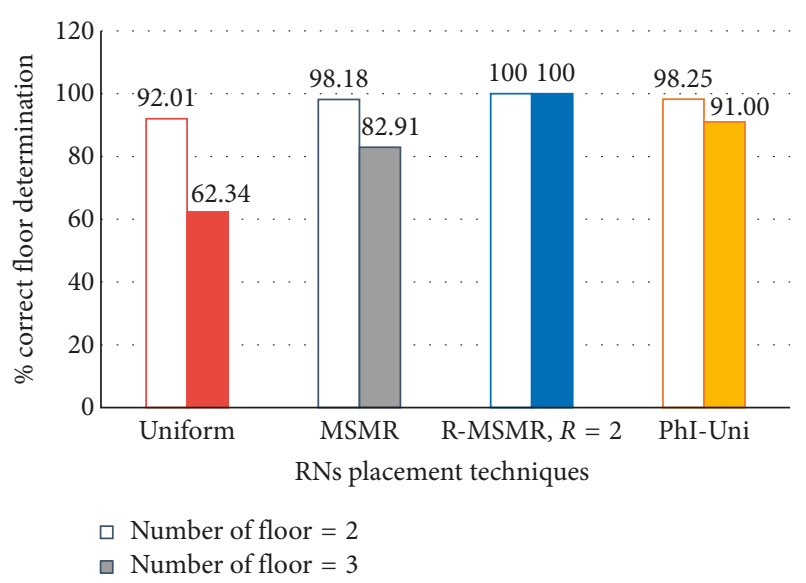

FIGURE 9: Percentage of the correct floor determination under three $\mathrm{RN}$ failure per floor.

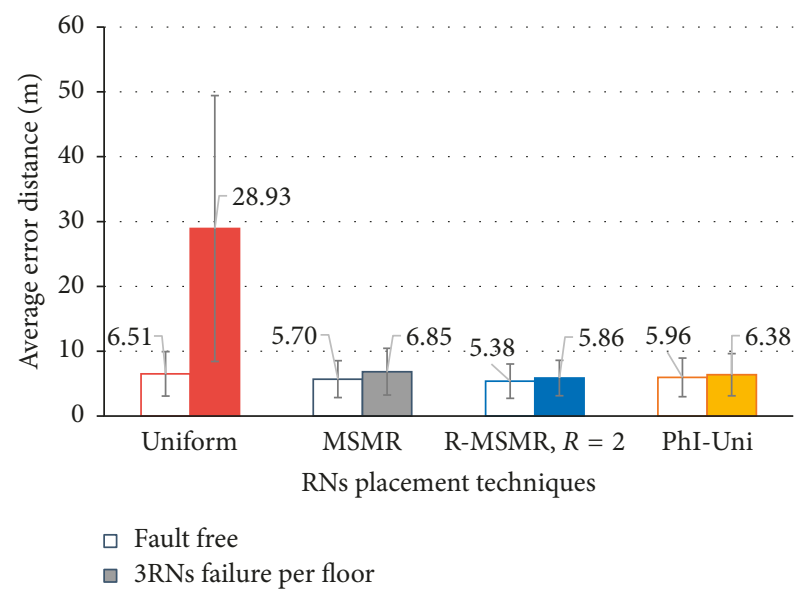

FIGURE 10: An average error distance of wireless indoor positioning systems in Scenario 1 (1 floor).

shows a better performance for location estimation than the Uniform by about $17.4 \%$ to $32.8 \%$, the MSMR by about $5.6 \%$ to $15.0 \%$, and the PhI-Uni by about $9.8 \%$ to $13.8 \%$. Additionally, we found that the accuracy performance trend for all four system designs under the fault-free scenario follows the increasing number of RNs installed in the service areas. A larger number of RNs installed can improve the location accuracy performance. Notice that this follows the same trend as in the performance improvement recommendations by Kaemarungsi [8].

We next consider the performance of the indoor positioning systems under the $3 \mathrm{RN}$ failure per floor scenario. Figure 13 shows an example of the cumulative density function (CDF) of the error distance for the two-floor service area of Scenario 2. The red lines, the black lines, the blue lines, and the yellow lines represent the precision performance of the Uniform, the MSMR, the R-MSMR with $R=2$, and the PhI-Uni, respectively. The results indicate that faulty RNs in the system decrease the positioning performance of all system designs. However, the R-MSMR with $R=2$ also achieves the highest positioning performance, in which the

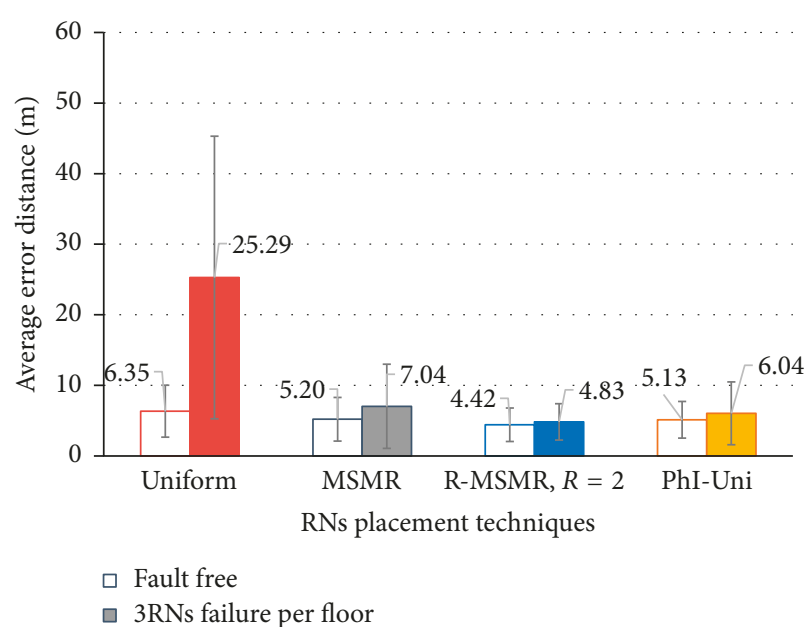

FIGURE 11: An average error distance of wireless indoor positioning systems in Scenario 2 (2 floors).

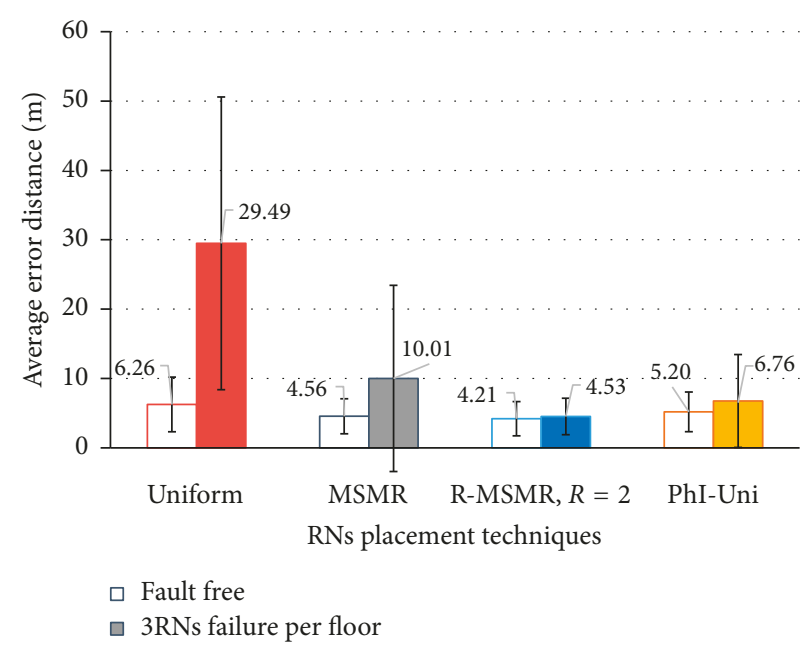

FIGURE 12: An average error distance of wireless indoor positioning systems in Scenario 3 (3 floors).

performance under the $\mathrm{RN}$-failure scenario is approximately $90 \%$ precision within 8.1 meters. This represents an $85.9 \%$ better performance than the Uniform (i.e., 90\% precision within 57.7 meters), $29.6 \%$ better performance than the MSMR (i.e., 90\% precision within 11.5 meters), and $11.4 \%$ better performance than the PhI-Uni (i.e., 90\% precision within 9.14 meters).

Considering the overall accuracy performance under the $3 R N$-failure per floor scenario, Figures 10-12 show that the R-MSMR with $R=2$ provides a better accuracy performance than the Uniform by about $79.7 \%$ to $84.6 \%$, the MSMR by about $14.5 \%$ to $54.7 \%$, and the PhI-Uni by about $8.2 \%$ to $32.9 \%$. Furthermore, the fault tolerance of the proposed $\mathrm{R}$-MSMR under all of the $\mathrm{RN}$-failure scenarios is greater than that of the other system designs. For instance, Figure 12 shows the positioning performance under the scenario of nine RN failures inside the three-floor service area. The R-MSMR with $R=2$ has a percentage of difference between 


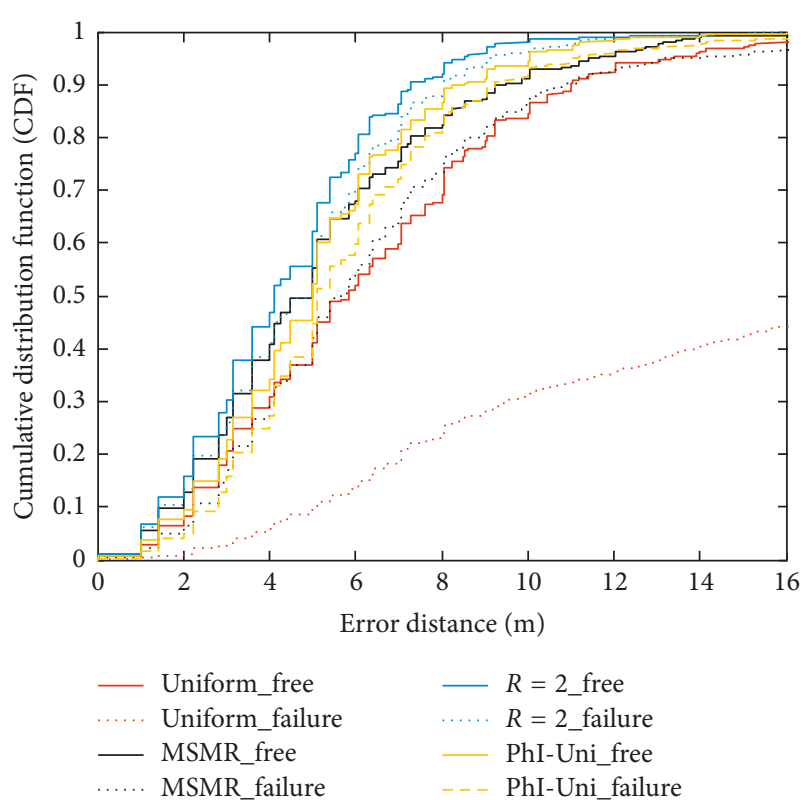

FIgURE 13: CDF of error distance in Scenario 2.

an average error distance in the normal situation and the $3 \mathrm{RN}$-failure per floor scenario of about $7.1 \%$, whereas the Uniform, the MSMR, and the PhI-Uni have percentage of up to $79 \%, 55 \%$, and $23.1 \%$, respectively. Although the number of RNs installed in the PhI-Uni is the same as the number of RNs installed in the proposed R-MSMR, the indoor positioning performances are different. The PhI-Uni only provides a correct floor determination score of $91 \%$ with a fault tolerance of $76.9 \%$, while the proposed R-MSMR achieves a successful correct floor determination score of $100 \%$ with a fault tolerance of $92.9 \%$. The reason why the proposed R-MSMR with $R=2$ can provide better fault tolerance during the online estimation phase than the other system designs is that the proposed R-MSMR is specifically designed to guarantee that the localization area (i.e., all STPs) must be able to receive at least the recommended number of RSS values with a summation of the accuracy index $(\alpha)$ and the reliability index $(R)$. Thus, the structure of the proposed system design can tolerate the missing RSS values during the online estimation phase where some RNs in the system fail. This ensures good positioning accuracy during a normal situation and yields the robustness of the location estimation under the scenario of RN failures. Furthermore, the sufficient number of RNs $\left(N_{S}\right)$ that is obtained from Phase I can also prevent the high cost of node infrastructure installation. Thus, the designers can apply the determining optimal number of RNs obtained from Phase I to place the RNs manually in a small service area or an uncomplicated building.

Figure 14 illustrates the performance improvement of the R-MSMR when the value of the reliability index is increased while the number of faulty RNs is fixed at six nodes (i.e., 3RN-failure per floor inside a two-floor service area). In Figure 14, four of the reliability indices for the R-MSMR are compared, specified as $R=1$ to $R=4$. The results show that a larger value for the reliability index can improve the

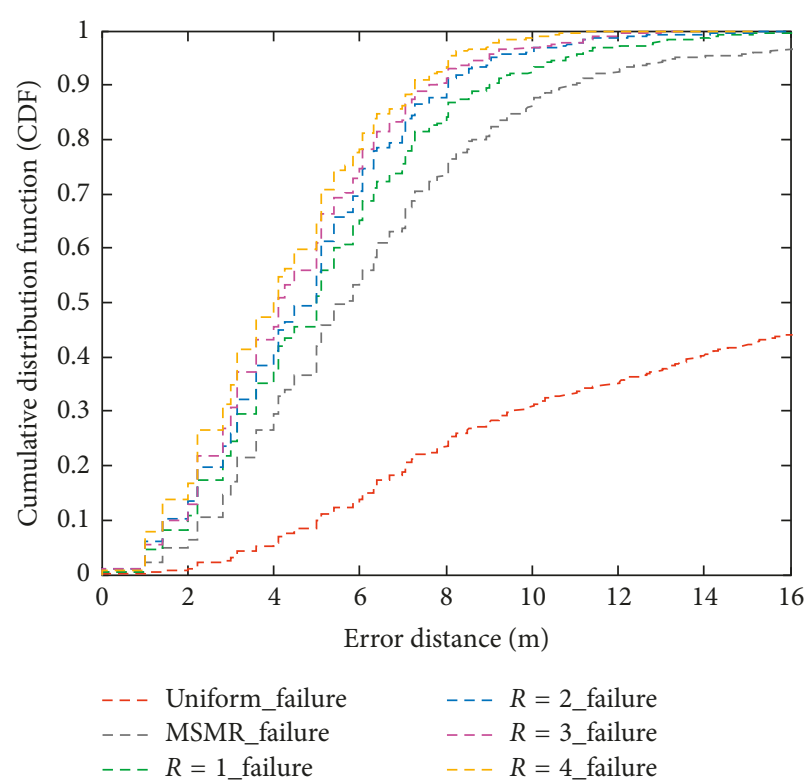

FIgURE 14: Effect of the reliability index on the error distance distribution (CDF) in Scenario 2.

positioning performance of the location estimation. For example, under the $3 \mathrm{RN}$-failure per floor scenario, the precision performance at 5 meters of the R-MSMR with $R=1$ to $R=4$ is approximately $51.1 \%, 55.6 \%, 61.1 \%$, and $65.3 \%$, respectively. This demonstrates that the system can handle the faulty RNs by utilizing the R-MSMR with the large value of its reliability index. However, increasing the reliability index for the R-MSMR requires a greater number of RNs to be installed in the system. This leads to higher installation costs, computation time, and memory space requirement for the fingerprint database. These factors will depend on the limitations of the indoor positioning systems chosen, such as the budget for installing the wireless network and the performance requirements of the positioning application.

5.3. Impact of RN-Failure Patterns on Accuracy Performance. In this section, we analyze the impact of different RN-failure patterns on accuracy performance. The system design results of the Uniform, the MSMR, the R-MSMR with $R=2$, and the PhI-Uni under the two-floor service area were considered, in which those RN-placement designs are as shown in Figure 7. The experimental settings in this study were assigned the same configuration as in Scenario 2. The RMoS floor algorithm and the active Euclidean distance technique were used to estimate the target locations. In this study, two different RN-failure patterns were considered. The first pattern was a similar RN-failure pattern, in which all RNs on the 1st floor and the 2 nd floor fail on the same side of the building. The second pattern involved an across $R N$-failure pattern, in which all faulty RNs on the 1st floor were on the opposite side of the building to the faulty RNs on the 2nd floor. In each RN-failure pattern, the four cases of the faulty RNs inside the building were divided 

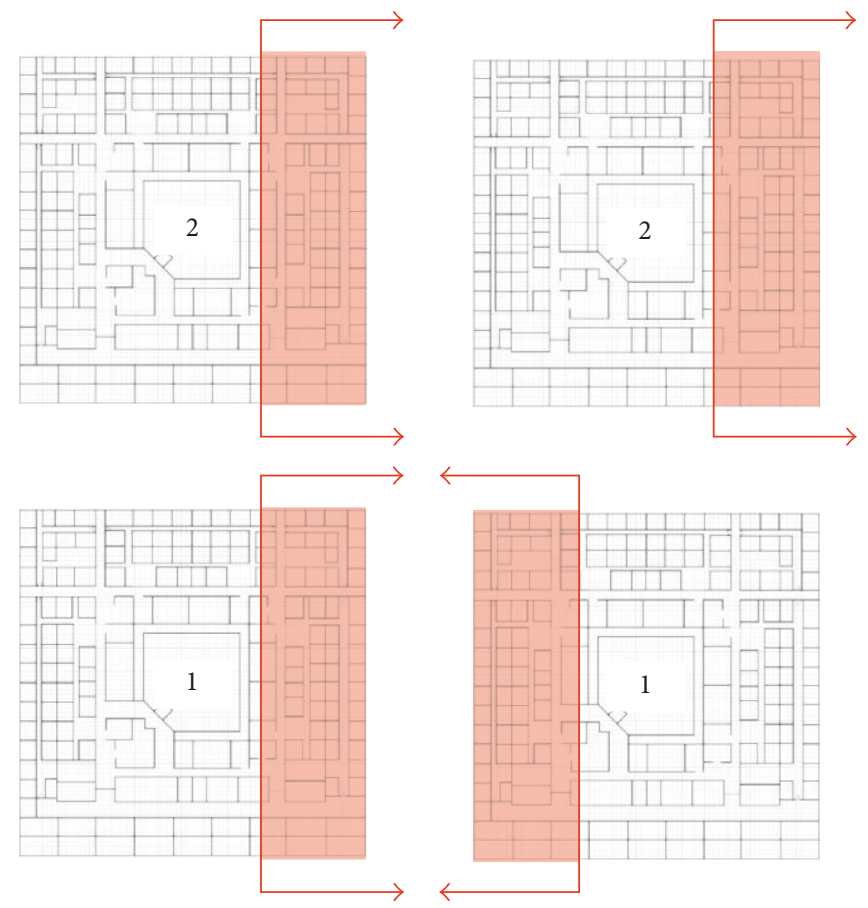

(a)

(b)

FIgURE 15: Example of the faulty RNs pattern in this experiment. (a) Similar RN-failure pattern. (b) Across RN-failure pattern.

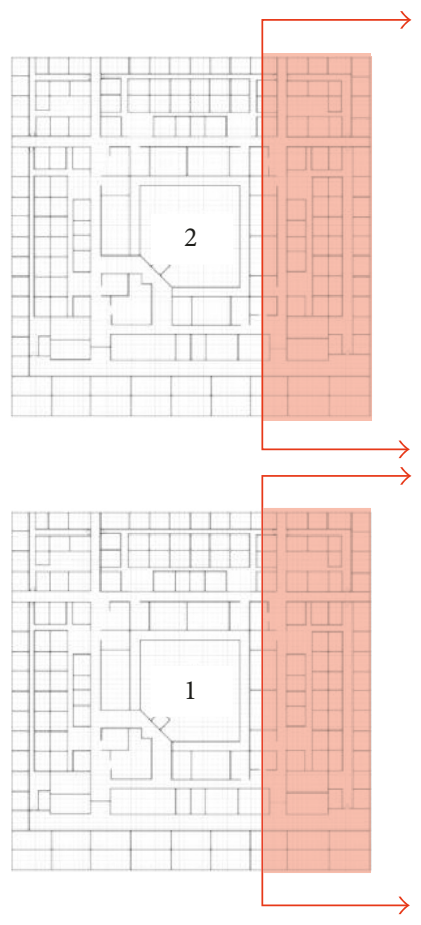

(a)
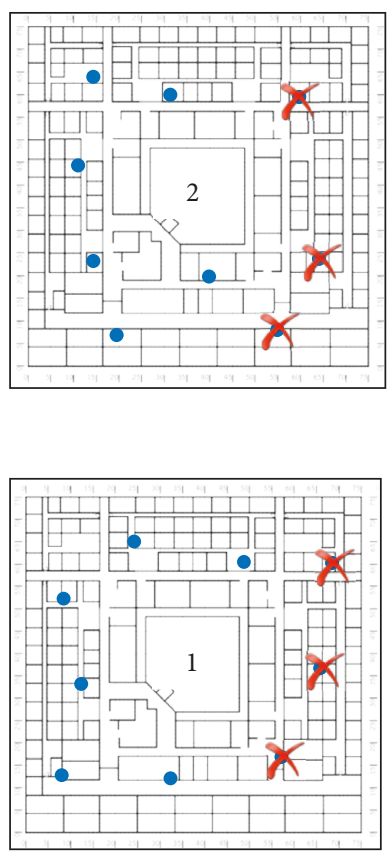

(b)

FIGURE 16: Example of the 1st similar RN-failure scenario for the R-MSMR, $R=2$.

according to the cardinal directions (i.e., north, south, west, and east direction). Figures 15(a) and 15(b) show an example of the 1st similar and the 1st across $\mathrm{RN}$-failure pattern, respectively. In particular, the six cases of the
$\mathrm{RN}$-failure per floor situations, consisting of from zero nodes to five nodes, are compared.

Figure 16 shows an example of indoor positioning systems in the two-story building that employs R-MSMR 


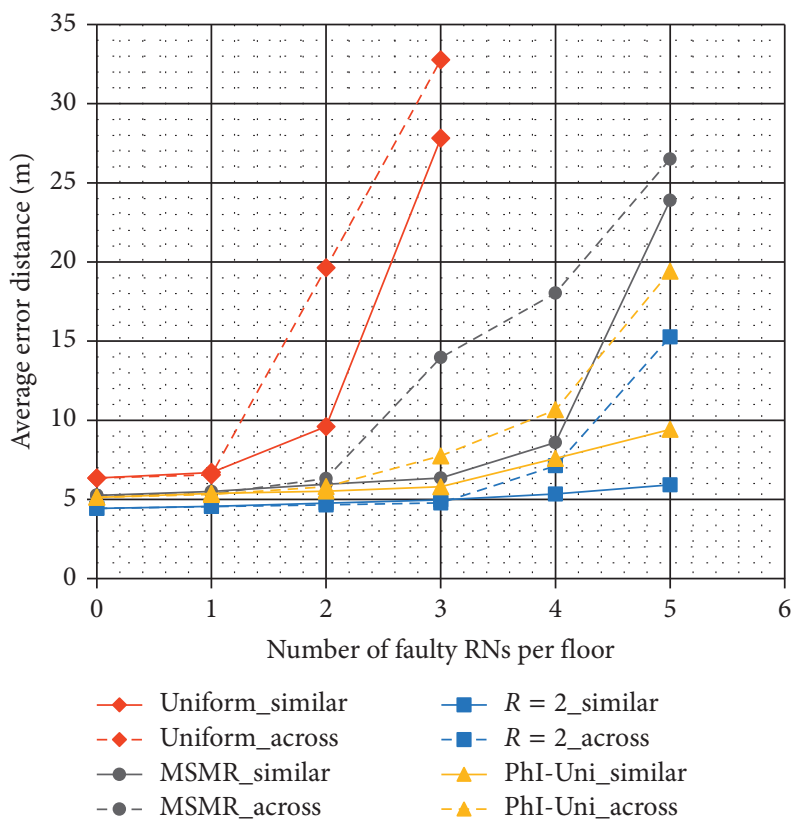

FIGURE 17: Effect of different numbers of RN failures per floor on average error distance.

with $R=2$. The blue circles represent the location of the RNs installed, while the red crosses represent the location of the $\mathrm{RN}$ failures. In this figure, it can be seen that the system encountered the 1st similar RN-failure pattern, in which three RNs on each floor fail. Those faulty RNs on each floor are located in the east section of the service area.

We first consider the impact of the number of $\mathrm{RN}$ failures on performance accuracy in Figure 17. The results of each system designs were averaged from the four $\mathrm{RN}$-failure sections (i.e., the four cardinal directions). Clearly, performance accuracy decreases in line with an increase in the number of RN failures. For example, Figure 17 shows the trend of the average error distance when the number of faulty RNs in each floor is increased. The MSMR under the similar RN-failure pattern (the black solid line with circles) has an increasing average error distance of 5.25, 5.50, 5.93, $6.34,8.59$, and 23.88 meters for $0,1,2,3,4$, and 5 failed RNs on each floor, respectively. This indicates that the accuracy performance of the MSMR drops by almost $80 \%$ if ten RNs inside the two-floor service area fail. These results are different from those of the proposed R-MSMR with $R=2$. Our proposed design can provide a better fault tolerance than other system designs. In this case, under the ten $\mathrm{RN}$ failure inside the two-floor service area scenario, the average error distance of the R-MSMR with $R=2$ does not exceed 6 meters.

Moreover, we found that the across RN-failure pattern (the dashed lines) degrades accuracy performance more than the similar RN-failure pattern (the solid lines). For example, the MSMR under the across RN-failure pattern (black dashed line with circles) has an increasing average error distance of $5.25,5.34,6.31,13.95,18.02$, and 26.49 meters for $0,1,2,3,4$, and 5 failed RNs on each floor, respectively. It resulted in a maximum of $52.4 \%$ worse location accuracy than the similar RN-failure pattern. This means that the
MSMR may have poor positioning performance if the across $\mathrm{RN}$-failure pattern occurs. Similar results were obtained for the cases of the Uniform, the R-MSMR with $R=2$, and PhIUni design under the across $\mathrm{RN}$-failure pattern.

Next, to investigate how the patterns of RN failure influence accuracy performance, we report the location estimation error performance of the similar and the across $\mathrm{RN}$-failure patterns as shown in Figures 18 and 19, respectively. In each figure, the indoor two-floor positioning systems that employ the Uniform and the R-MSMR with $R=2$ were compared under the case of the two RN failure per floor scenario. The pink lines denote the error distance that is greater than eight meters. The blue circles represent the actual target locations, while the pink crosses represent the estimated target locations. We first consider indoor positioning systems under the similar RN-failure pattern as shown in Figure 18. Clearly, the Uniform has higher location estimation error than the R-MSMR with $R=2$. The average error distance of the Uniform is 10.33 meters, while the average error distance of the R-MSMR with $R=2$ is 4.71 meters. Moreover, Figure 18(a) indicates that most of the location estimation errors for the Uniform are located on the opposite side of the floor to the faulty RNs (i.e., at the west side of the building). These results are different from those of the R-MSMR with $R=2$. As shown in Figure 18(b), the R-MSMR with $R=2$ has $54.4 \%$ better accuracy performance than the Uniform. Note that both the Uniform and the R-MSMR with $R=2$ can achieve $100 \%$ correct floor determination in the case of the similar $\mathrm{RN}$-failure pattern.

We next consider the indoor positioning systems under the across RN-failure pattern, as shown in Figure 19. The results indicate that the Uniform again has higher location estimation error than the proposed R-MSMR with $R=2$. Those location estimation errors are mostly located on the opposite side of the floor to the faulty RNs. Besides the issue 

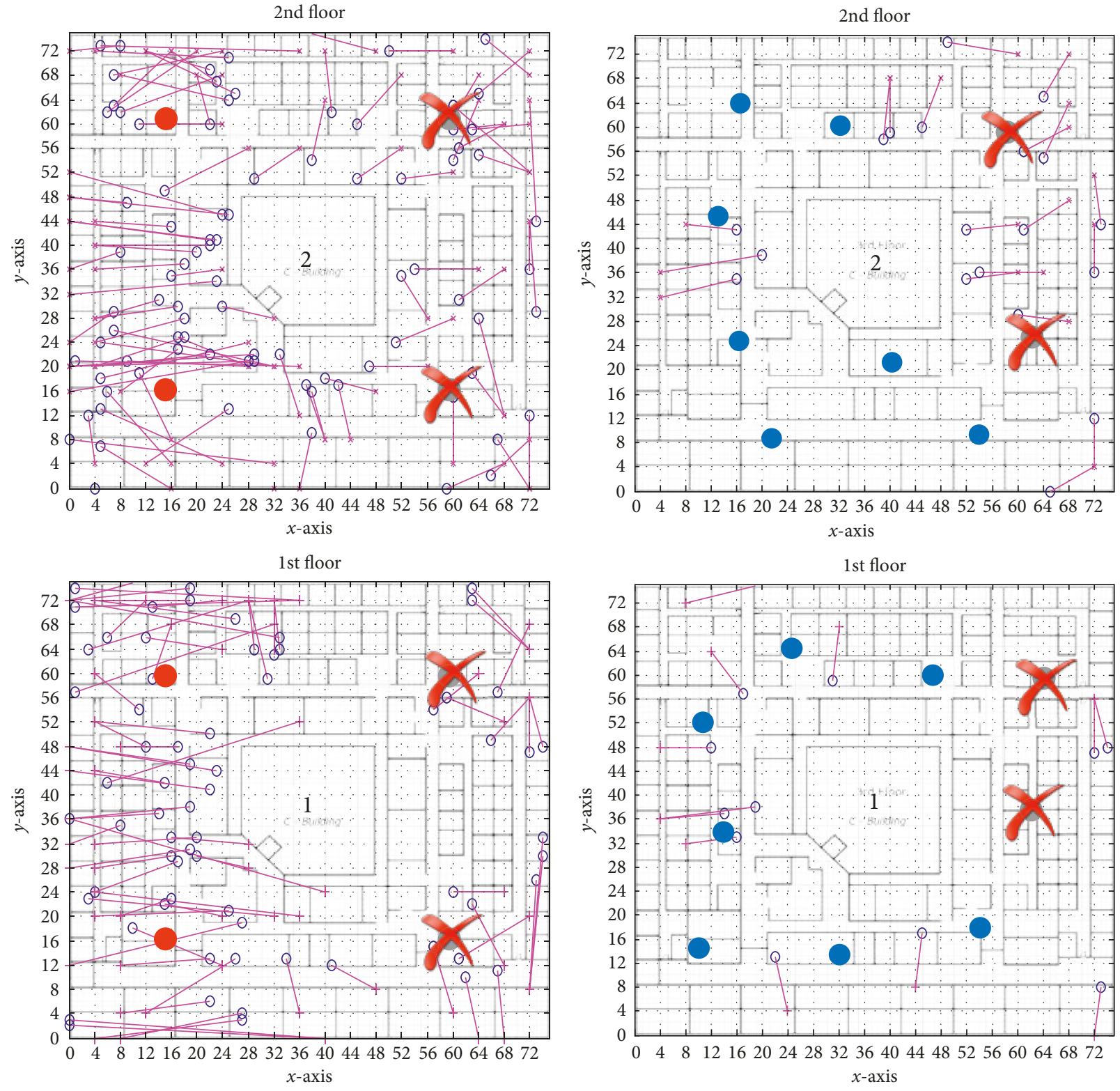

(a)

(b)

FIgURE 18: The location estimation errors that are greater than 8 meters under the similar RN-failure pattern. (a) The Uniform. (b) The R-MSMR, $R=2$.

of location estimation errors, incorrect floor determination also occurred under the across RN-failure pattern. In Figure 19(a), the red solid lines represent the results of the error distance when estimating the incorrect floor determination. The Uniform shows the lowest floor accuracy performance under the across RN-failure pattern (with an average error distance of 20.3 meters). The Uniform provides $74.0 \%$ correct floor determination, with most of the floor determination errors located on the same side of the floor as the faulty RNs. These results are different from those of the proposed system design. The R-MSMR with $R=2$ also has an average error distance of 4.72 meters, but it yields $100 \%$ correct floor determination. Once again, the overall accuracy performance of the Uniform under the across $\mathrm{RN}$-failure pattern was up to $49.1 \%$ worse than the location accuracy for the similar RN-failure pattern, whereas the overall accuracy performance of the proposed R-MSMR with $R=2$ was almost the same under both the similar and the across RNfailure patterns.

The results show the advantages of the proposed robust system design, which not only provides the highest performance accuracy during a normal situation, but also yields reliable location results under unexpected situations such as RN failures. The reason for this is that the mathematical formulation of the proposed R-MSMR model considers not only the radio signal coverage requirements but also the accuracy and reliability requirements. Unlike the proposed system design, existing RN-placement designs limit their 

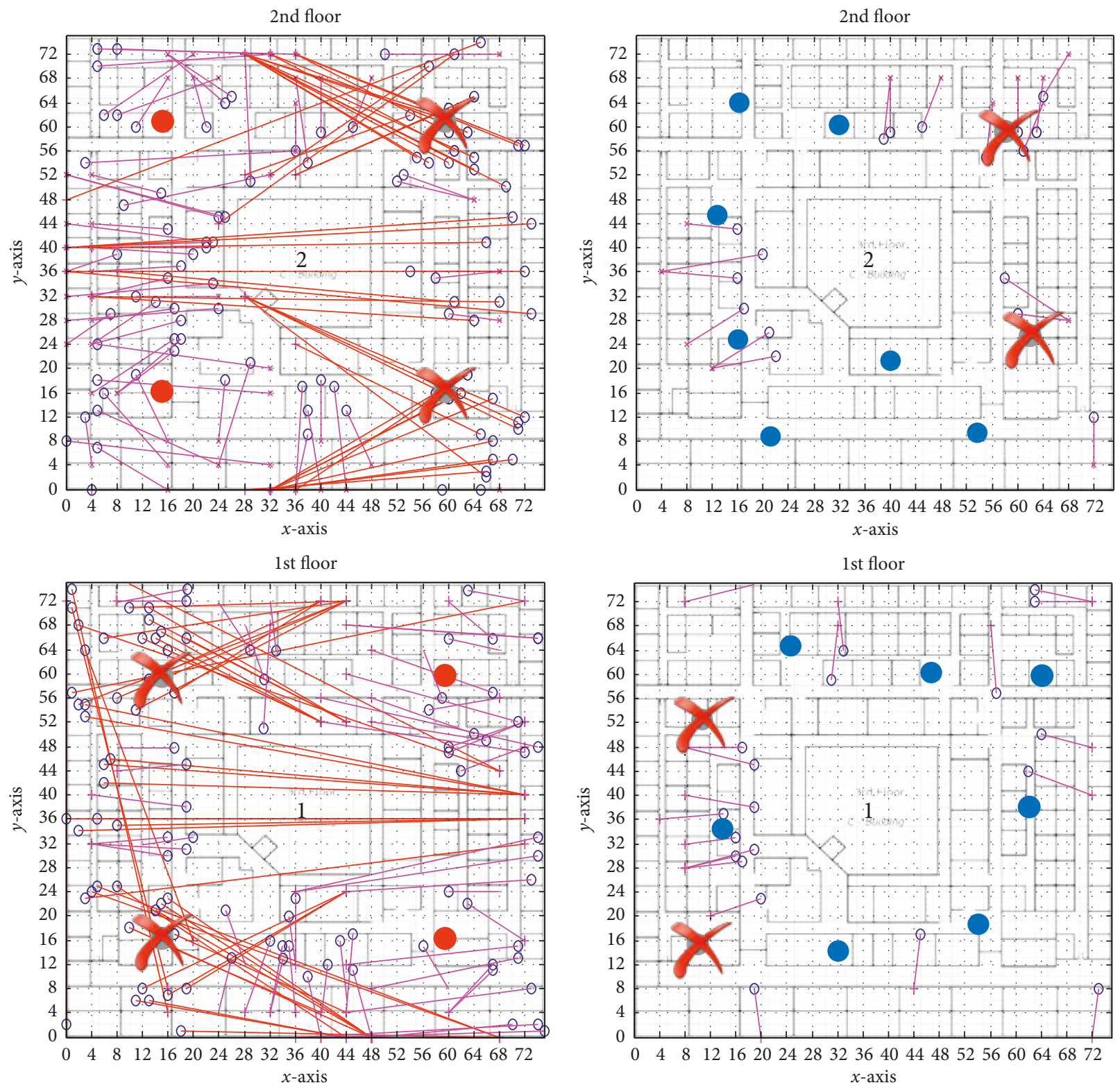

(a)

(b)

FIgURE 19: The location estimation errors that are greater than 8 meters under the across RN-failure pattern. (a) The Uniform. (b) The RMSMR, $R=2$.

focuses to the provision of signal radio coverage in the service area. These are insufficient and inefficient methods for designing indoor positioning systems in unexpected situations. Thus, we can conclude that an indoor positioning systems that employ our proposed R-MSMR is fault tolerant and robust in the $\mathrm{RN}$-failure scenarios considered in our study.

\section{Conclusion}

In this article, we have considered the problem of wireless indoor positioning system design, for systems based on the location fingerprinting technique. We proposed a novel mathematical formulation using a Binary Integer Linear Programming (BILP) approach that employs Simulated
Annealing (SA) based on the heuristic solution technique. The task of the proposed system design is to determine and place a suitable number of RNs and to find the optimum locations which may be located on a single floor or on multiple floors. In particular, the proposed system design has been developed to support robust operation both during a normal situation and when some RNs have failed. Experimental results reveal that the proposed robust system design outperformed other system designs and was able to achieve the highest location performance accuracy in both fault-free and $R N$-failure scenarios. Specifically, the proposed system design delivered $84.6 \%, 54.7 \%$, and $32.9 \%$ better performance accuracy than the Uniform, the MSMR, and the PhI-Uni, respectively. 
Our future works will consider a combination of the major components between the robust system design and the robust localization algorithm that may be supported during the normal situation or when some RNs have failed.

\section{Conflicts of Interest}

The authors declare that they have no conflicts of interest.

\section{Acknowledgments}

The authors would like to express their deepest appreciation to the late Dr. Chutima Prommak, Assistant Professor of the School of Telecommunication Engineering at Suranaree University of Technology, Thailand, whose contribution to this work was of great significance. Without her experience, guidance, and patience, it would never have been possible to complete this work successfully.

\section{References}

[1] A. Gharaibeh, M. A. Salahuddin, S. J. Hussini et al., "Smart cities: a survey on data management, security and enabling technologies," IEEE Communications Surveys \& Tutorials, vol. 19, no. 4, pp. 2456-2501, 2017.

[2] C. C. Li, J. Su, and E. T.-H. Chu, "Building/environment data/information enabled location specificity and indoor positioning," IEEE Internet of Things Journal, vol. 4, no. 6, pp. 2116-2128, 2017.

[3] A. A. Nazari, A. Yassine, and S. Shirmohammadi, "Equipment location in hospitals using RFID-based positioning system," IEEE Transactions on Information Technology in Biomedicine, vol. 16, no. 6, pp. 1058-1069, 2012.

[4] Y.-C. Wang and C.-C. Yang, "3s-cart: a lightweight, interactive sensor-based cart for smart shopping in supermarkets," IEEE Sensors Journal, vol. 16, no. 17, pp. 6774-6781, 2016.

[5] K. Pahlavan and P. Krishnamurthy, Principles of Wireless Access and Localization, Wiley, Hoboken, NJ, USA, 2013.

[6] D. Dardari, P. Closas, and P. M. Djurić, "Indoor tracking: theory, methods, and technologies," IEEE Transactions on Vehicular Technology, vol. 64, no. 4, pp. 1263-1278, 2015.

[7] R. S. Campos, L. L. Marcello, and L. R. Campos, "Wi-Fi multifloor indoor positioning considering architectural aspects and controlled computational complexity," Expert Systems with Applications, vol. 41, no. 14, pp. 6211-6223, 2014.

[8] K. Kaemarungsi, "Efficient design of indoor positioning systems based on location fingerprinting," in Proceedings of Wireless Networks Communications and Mobile Computing, pp. 181-186, Lahaina, HI, USA, June 2005.

[9] R. Akl, K. Pasupathy, and M. Haidar, "Anchor nodes placement for effective passive localization," in Proceedings of International Conference on Selected Topics in Mobile and Wireless Networking (iCOST), pp. 127-132, Shanghai, China, October 2011.

[10] O. Baala, Y. Zheng, and A. Caminada, "The impact of AP placement in WLAN-based indoor positioning system," in Proceedings of ICN '09 Eighth International Conference on Networks, pp. 12-17, Guadeloupe, France, March 2009.

[11] P. Sadhukhan, P. Sadhukhan, and Z. Pervez, "Impact of beacon coverage on clustering strategies for fingerprinting localization system," in Proceedings of International Conference on Computing, Networking and Communications (ICNC), 5 pages, Maui, Hawaii, USA, March 2017.
[12] R. Zhang, W. Xia, Z. Jia, L. Shen, and J. Guo, "The optimal placement method of anchor nodes toward RSS-based localization systems," in Proceedings of Sixth International Conference on Wireless Communications and Signal Processing (WCSP), pp. 1-6, Hefei, China, October 2014.

[13] S. Aomumpai, K. Kondee, C. Prommak, and K. Kaemarungsi, "Optimal placement of reference nodes for wireless indoor positioning systems," in Proceedings of 11th International Conference on Electrical Engineering/Electronics, Computer, Telecommunications and Information Technology, pp. 1-6, Nakhon Ratchasima, Thailand, July 2014.

[14] A. E. C. Redondi and E. Amaldi, "Optimizing the placement of anchor nodes in RSS-based indoor localization systems," in Proceedings of 12th Annual Mediterranean Ad Hoc Networking Workshop (MED-HOC-NET), pp. 8-13, Ajaccio, France, June 2013.

[15] S. Merkel, P. Unger, and H. Schmeck, "Evolutionary algorithm for optimal anchor node placement to localize devices in a mobile ad hoc network during building evacuation," in Proceedings of Genetic and Evolutionary Computation Conference (GECCO '13), pp. 1407-1414, New York, NY, USA, July 2013.

[16] G. Mao, B. Fidanb, and B. D. O. Anderson, "Wireless sensor network localization techniques," Computer Networks, vol. 51, no. 10, pp. 2529-2553, 2007

[17] C. Yang and H.-R. Shao, "WiFi-based indoor positioning," IEEE Communications Magazine, vol. 53, no. 3, pp. 150-157, 2015.

[18] S. He and S.-H. G. Chan, "Wi-Fi fingerprint-based indoor positioning: recent advances and comparisons," IEEE Communications Surveys \& Tutorials, vol. 18, no. 1, pp. 466-490, 2015.

[19] Q. Wang, Y. Feng, X. Zhang, Y. Sun, and X. Lu, "IWKNN: an effective Bluetooth positioning method based on isomap and WKNN," Mobile Information Systems, vol. 2016, Article ID 8765874, 11 pages, 2016.

[20] P. Kriz, F. Maly, and T. Kozel, "Improving indoor localization using Bluetooth low energy beacons," Mobile Information Systems, vol. 2016, Article ID 2083094, 11 pages, 2016.

[21] S.-H. Fang and T.-N. Lin, "Indoor location system based on discriminant-adaptive neural network in IEEE 802.11 environments," IEEE Transactions on Neural Networks, vol. 19, no. 11, pp. 1973-978, 2008.

[22] K. Kaemarungsi, "Distribution of WLAN received signal strength indication for indoor location determination," in Proceedings of 1st International Symposium on Wireless Pervasive Computing, 5 pages, Phuket, Thailand, January 2006.

[23] J. Chen, Y. Ye, and K. Pahlavan, "UWB characteristics of creeping wave for RF localization around the human body," in Proceedings of IEEE 23rd International Symposium on Personal Indoor and Mobile Radio Communications (PIMRC), pp. 1290-294, Sydney, Australia, September 2012.

[24] Q. Li, W. Li, W. Sun, J. Li, and Z. Liu, "Fingerprint and assistant nodes based Wi-Fi localization in complex indoor environment," IEEE Access, vol. 4, pp. 2993-3004, 2016.

[25] T. Sathyan and M. Hedley, "Fast and accurate cooperative tracking in wireless networks," IEEE Transactions on Mobile Computing, vol. 12, no. 9, pp. 1801-1813, 2012.

[26] Y. Qian, K. Lu, and D. Tipper, "A design for secure and survivable wireless sensor networks," IEEE Wireless Communications, vol. 14, no. 5, pp. 30-37, 2007.

[27] C. Khauphung, P. Keeratiwintakorn, and K. Kaemarungsi, "On robustness of centralized-based location determination using WSN," in Proceedings of 14th Asia-Pacific Conference 
on Communications (APCC), pp. 1-5, Tokyo, Japan, October 2009.

[28] N. Correia, J. Coimbra, and G. Schütz, "Fault-tolerance planning in multiradio hybrid wireless-optical broadband access networks," IEEE/OSA Journal of Optical Communications and Networking, vol. 1, no. 7, pp. 645-654, 2009.

[29] T. Liu, W. Yang, and R. Bu, "Reliable telecommunication network design problem under node failure," in Proceedings of 11th International Symposium on Operations Research and its Applications in Engineering, Technology and Management 2013 (ISORA 2013), pp. 1-8, Huangshan, China, August 2014.

[30] J. Luo, W. Wu, and M. Yang, "Interference-aware gateway placement for wireless mesh networks with fault tolerance assurance," in Proceedings of IEEE International Conference on Systems Man and Cybernetics (SMC), pp. 2373-2380, Istanbul, Turkey, October 2010.

[31] Y. Zhang and W. Ye, "Design and placement of light monitoring system in museums based on wireless sensor networks," in Proceedings of International Symposium on Advanced Control of Industrial Processes (ADCONIP), pp. 512-517, Hangzhou, China, May 2011.

[32] C. Sharma, Y. F. Wong, W.-S. Soh, and W.-C. Wong, "Access point placement for fingerprint-based localization," in Proceedings of IEEE International Conference on Communication Systems (ICCS), pp. 238-243, Singapore, November 2011.

[33] S.-H. Fang and T.-N. Lin, "A novel access point placement approach for WLAN-based location systems," in Proceedings of IEEE Wireless Communications and Networking Conference (WCNC), pp. 1-4, Sydney, Australia, April 2010.

[34] K. Kondee, S. Aomumpai, and C. Prommak, "A novel technique for reference node placement in wireless indoor positioning systems based on fingerprint technique," ECTI Transactions on Computer and Information Technology (ECTI-CIT), vol. 9, no. 2, pp. 131-141, 2015.

[35] W. L. Winston and J. B. Goldberg, Operations Research: Applications and Algorithms, Thomson Brooks/Cole, Pacific Grove, CA, USA, 2004.

[36] S. Kirkpatrick, C. D. Gelatt, and M. P. Vecchi, "Optimization by simulated annealing," Science, vol. 220, no. 4598, pp. 671-680, 1983.

[37] K. Maneerat, K. Kaemarungsi, and C. Prommak, "Robust floor determination algorithm for indoor wireless localization systems under reference node failure," Mobile Information Systems, vol. 2016, Article ID 4961565, 12 pages, 2016.

[38] R. Jain, The Art of Computer Systems Performance Analysis: Techniques for Experimental Design, Measurement, Simulation, and Modeling, Wiley, Hoboken, NJ, USA, 1st edition, 1991.

[39] MC13224V: 2.4 GHz 802.15.4 RF and 32-bit ARM $7^{\mathrm{TM}} M C U$ with $128 \mathrm{~KB}$ flash, 96KB RAM, NXP Semiconductors, http:// www.nxp.com/products/wireless-connectivity/2.4-ghz-wirelesssolutions/2.4-ghz-802.15.4-rf-and-32-bit-arm7-mcu-with-128kbflash-96kb-ram:MC13224V, 2010. 


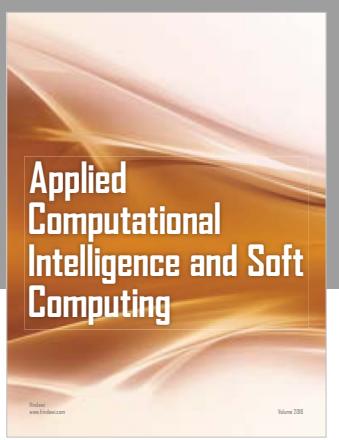

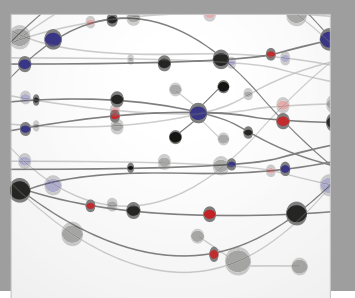

The Scientific World Journal
Submit your manuscripts at

Computing
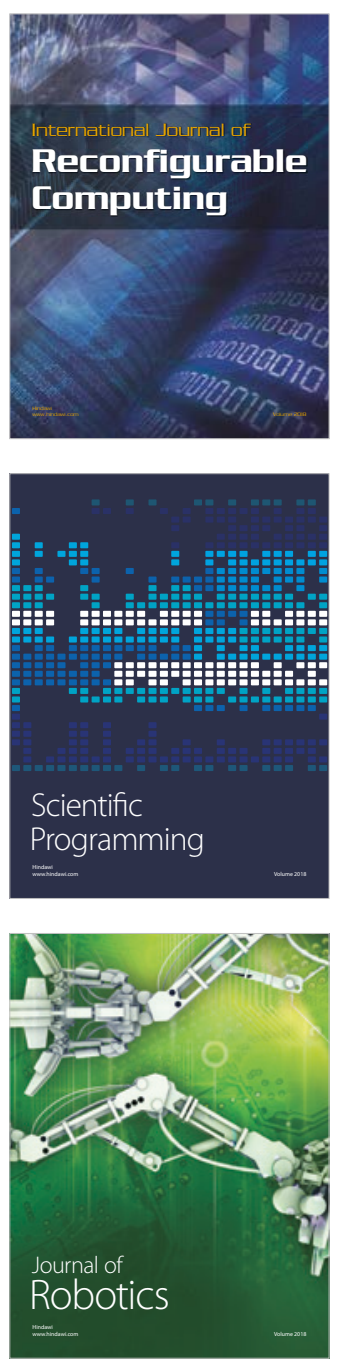

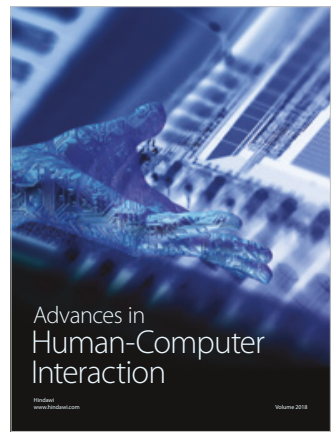

Human-Compute

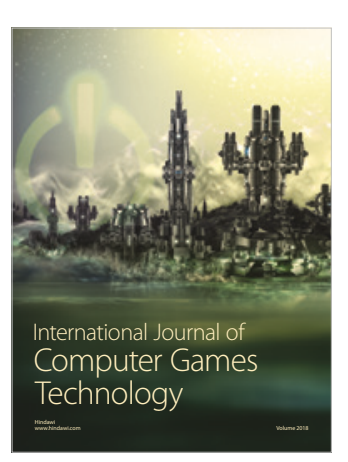

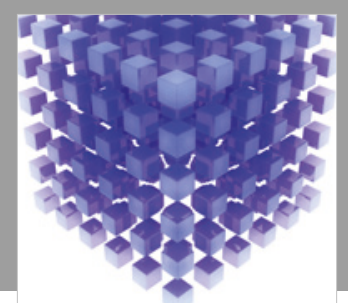

Mathematical Problems in Engineering

\section{Engincering}
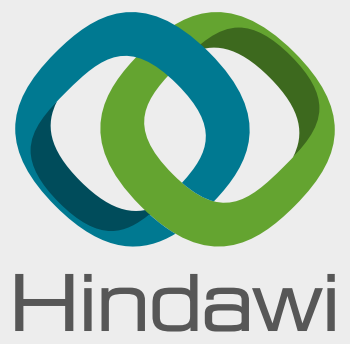

www.hindawi.com
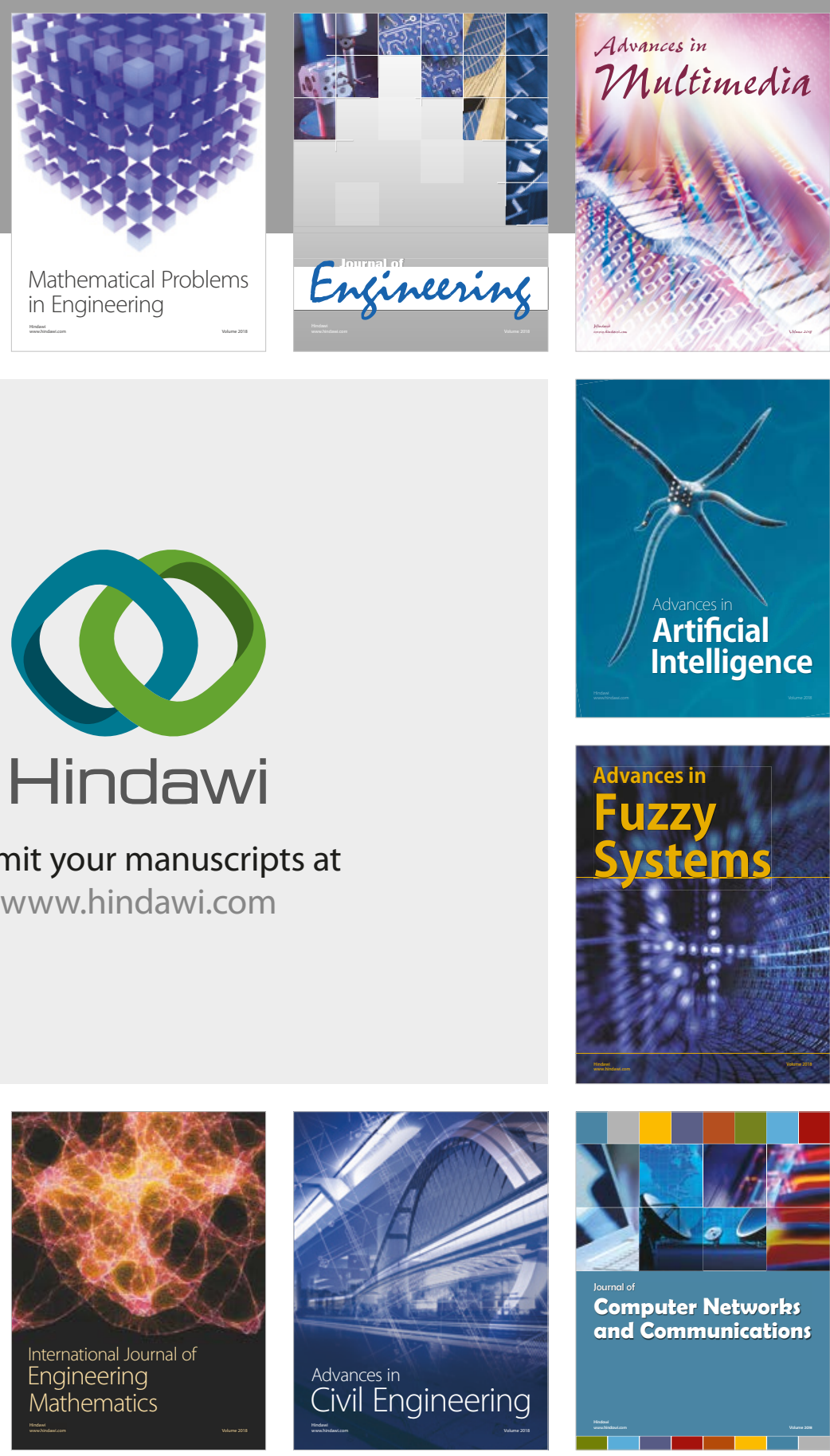

Computer Networks and Communications

Multimedia
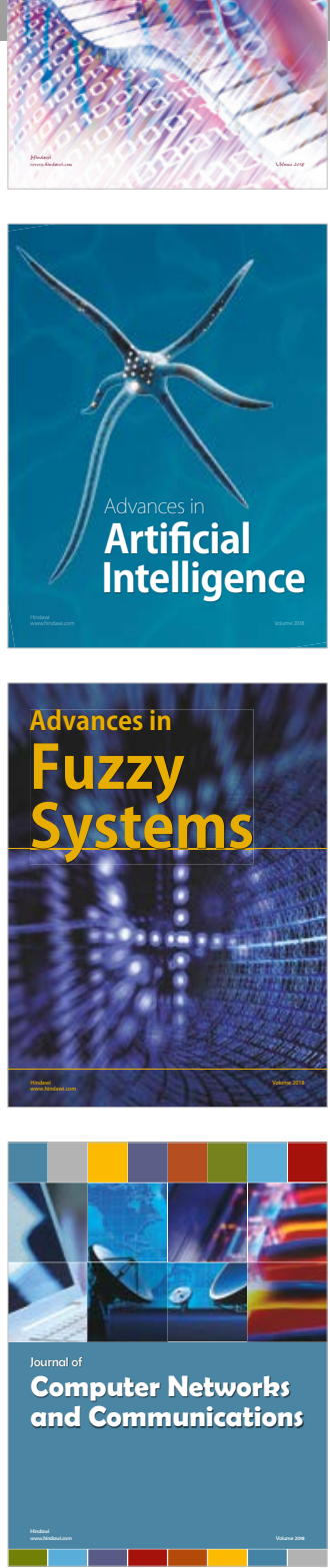

Advances in

Modelling \&

Simulation

in Engineering

interaction

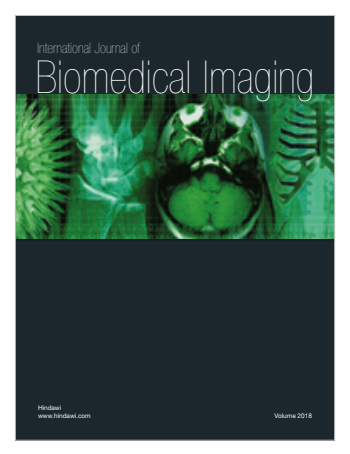

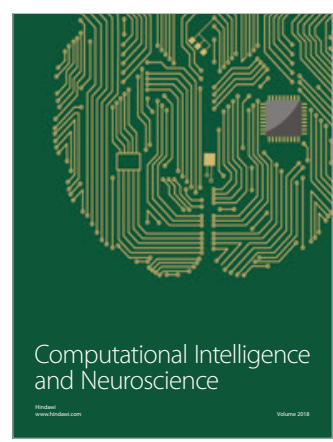

